\title{
Specific genetic markers for detecting subtypes of dengue virus serotype-2 in isolates from the states of Oaxaca and Veracruz, Mexico
}

\author{
Catalina E Gardella-Garcia ${ }^{\dagger 1}$, Gerardo Perez-Ramirez ${ }^{\dagger 2}$, Joel Navarrete- \\ Espinosa $^{3}$, Alejandro Cisneros ${ }^{4}$, Fabiola Jimenez-Rojas ${ }^{5}$, Luis R Ramírez- \\ Palacios $^{5}$, Rocio Rosado-Leon ${ }^{6}$, Minerva Camacho-Nuez ${ }^{2}$ and Maria \\ de L Munoz*1
}

Address: ${ }^{1}$ Department of Genetics and Molecular Biology, Centro de Investigacion y de Estudios Avanzados del Instituto Politecnico Nacional, Av. Instituto Politecnico Nacional 2508, San Pedro Zacatenco, C.P. 07360, Mexico D. F., Mexico, ${ }^{2}$ Program of Genomic Sciences, Universidad Autonoma de la Ciudad de Mexico, San Lorenzo \#290 Col. Del Valle, Mexico D. F., Mexico, ${ }^{3}$ Division de Epidemiologia, Coordinacion de Programas Integrados de Salud, Instituto Mexicano del Seguro Social. Av. Insurgentes Sur 253, $7^{\circ}$ Piso, Col. Roma, C.P. 06700 Mexico D. F. Mexico, ${ }^{4}$ Escuela de Medicina Veterinaria y Zootecnia, Universidad Autonoma Benito Juarez de Oaxaca, Oaxaca, Mexico, ${ }^{5}$ Laboratorio Estatal de Salud Publica de Oaxaca, Servicios de Salud de Oaxaca, Oaxaca, Mexico and ${ }^{6}$ Delegacion Veracruz Norte. IMSS, Veracruz, Mexico

Email: Catalina E Gardella-Garcia - evgardella@yahoo.com.mx; Gerardo Perez-Ramirez - geraperami@gmail.com; Joel NavarreteEspinosa - joel.navarrete@imss.gob.mx; Alejandro Cisneros - acisneross@gmail.com; Fabiola Jimenez-Rojas - fajiro1@hotmail.com; Luis R Ramírez-Palacios - acisneross@gmail.com; Rocio Rosado-Leon - rocio.rosado@imss.gob.mx; Minerva CamachoNuez-mcamachonuez@yahoo.com.mx; Maria de L Munoz*-lmunoz@cinvestav.mx

* Corresponding author †Equal contributors

Published: 15 July 2008

BMC Microbiology 2008, 8:117 doi:10.1186/1471-2180-8-117

This article is available from: http://www.biomedcentral.com//47/-2/80/8/II7

(C) 2008 Gardella-Garcia et al; licensee BioMed Central Ltd.

This is an Open Access article distributed under the terms of the Creative Commons Attribution License (http://creativecommons.org/licenses/by/2.0), which permits unrestricted use, distribution, and reproduction in any medium, provided the original work is properly cited.
Received: 22 December 2007

Accepted: 15 July 2008

\begin{abstract}
Background: Dengue (DEN) is an infectious disease caused by the DEN virus (DENV), which belongs to the Flavivirus genus in the family Flaviviridae. It has a (+) sense RNA genome and is mainly transmitted to humans by the vector mosquito Aedes aegypti. Dengue fever (DF) and dengue hemorrhagic fever (DHF) are caused by one of four closely related virus serotypes (DENV-I, DENV-2, DENV-3 and DENV-4). Epidemiological and evolutionary studies have indicated that host and viral factors are involved in determining disease outcome and have proved the importance of viral genotype in causing severe epidemics. Host immune status and mosquito vectorial capacity are also important influences on the severity of infection. Therefore, an understanding of the relationship between virus variants with altered amino acids and high pathogenicity will provide more information on the molecular epidemiology of DEN. Accordingly, knowledge of the DENV serotypes and genotypes circulating in the latest DEN outbreaks around the world, including Mexico, will contribute to understanding DEN infections.
\end{abstract}

Results: I. We obtained 88 isolates of DENV, 27 from Oaxaca and 6I from Veracruz. 2. Of these 88 isolates, 16 were serotype I; 62 serotype 2; 7 serotype 3; and 2 serotype 4 . One isolate had 2 serotypes (DENV-2 and -I). 3. Partial nucleotide sequences of the genes encoding C- prM (I4 sequences), the NS3 helicase domain (7 sequences), the NS5 S-adenosyl methionine transferase domain (7 sequences) and the RNA-dependent RNA polymerase (RdRp) domain (I8 sequences) were obtained. Phylogenetic analysis showed that DENV-2 isolates belonged to the Asian/ American genotype. In addition, the Asian/American genotype was divided into two clusters, one 
containing the isolates from $200 \mathrm{I}$ and the other the isolates from 2005-2006 with high bootstrap support of $94 \%$.

Conclusion: DENV-2 was the predominant serotype in the DF and DHF outbreak from 2005 to 2006 in Oaxaca State as well as in the 2006 outbreak in Veracruz State, with the Asian/American genotype prevalent in both states. Interestingly, DENV-I and DENV-2 were the only serotypes related to DHF cases. In contrast, DENV-3 and DENV-4 were poorly represented according to epidemiological data reported in Mexico. We found that isoleucine was replaced by valine at residue 106 of protein $C$ in the isolates from these 2005-2006 outbreaks and in those from the 1997, 1998 and 2001 outbreaks in the Caribbean islands. We suggested that this amino acid change may be used as a signature for isolates arising in the Caribbean islands and pertaining to the Asian/ American genotype. Other amino acid changes are specific for the Asian/American, Asian and American strains.

\section{Background}

DF and DHF/DSS are mosquito-borne infectious diseases transmitted by the vector Ae. Aegypti and have become a major global health concern. This mosquito vector is endemic in several regions of Mexico, especially the South Pacific Coast [1-3]. DF and DHF/DSS occur in tropical and sub-tropical regions around the world, predominantly in urban and suburban areas. The illness is endemic in more than 100 cities in Africa, America, the Mediterranean region and Southeast Asia [4]. Fifty million people are infected every year; approximately 500,000 of them are hospitalized and between 5\% and 15\% die [5]. This viral illness is characterized by a sudden onset of symptoms including high fever, severe headaches, extreme myalgias and arthralgias, retro-orbital pain, anorexia, gastrointestinal disturbances, exanthema, nausea and vomiting; a rash may appear three or four days after the fever begins $[6,7]$.

The infection caused by any of the four serotypes of DENV confers specific permanent immunity to the virus but does not protect against the other serotypes [8]; however, shortterm cross-immunity against the other three serotypes has been reported [9]. People living in an endemic area can be infected with any of the four serotypes of DENV and can even be simultaneously infected by two serotypes [10]. The relevance to pathology of the serotype and genotype of the infecting strain has been described several times [11-13].

The increase in the number of DHF and DSS cases worldwide is not fully understood. It was first explained by mutations making DENV more virulent, but this hypothesis has not been supported. Then, the "secondary infection" or "immune enhancement" hypothesis was proposed to explain the increased virulence of DENV in patients with secondary infections. This hypothesis is still favoured [14]. However, observations in Southeast Asia, some Pacific islands and the Americas are not consistent with it, so it has been modified several times. Advances in molecular biology have led to the recognition that some viral strains are more virulent than others [11-13]. The introduction of DHF into Cuba in 1981 was linked to the arrival of a Southeast Asian strain of DENV-2 on the island [15]. Subsequently, an extended outbreak of DHF/DSS occurred in Venezuela and Mexico, and this was linked to the introduction of the same Southeast Asian strain of DENV-2 [16,17]. Furthermore, a major epidemic of DF due to DENV-2 with the American genotype was reported in Peru in 1995, about 5 years after an epidemic of DENV1 [18]. The American DENV-2 genotype strains may have lacked the properties necessary to cause severe disease. The American genotype has been associated with mild disease (DF), while the Southeast Asian genotype coincided with the appearance of DHF on the American continent [11]. On this basis, viral virulence and immune responses have been considered two major factors in the pathogenesis of DHF.

Early phylogenetic studies organized DENV sequences into clusters on trees and classified them into a series of genotypes or subtypes [19-22]. Within DENV-2, six genotypes have been proposed, five of which occur in humans $[22,23]$. Although the Asian/American genotype originated in Southeast Asia and spread to the Americas in the early 1980s [22], the strains phylogenetically classified as Asian I and Asian II are currently restricted to Southeast Asia [24]; the American genotype is mostly found in the Americas and its frequency is now decreasing; and the Cosmopolitan genotype is widely distributed across the tropical and subtropical world [24,25]. DENV-1 consists of five subtypes (I-V) [22]. The DENV-3 and -4 viruses are currently classified into four and two genotypes, respectively $[19,20]$.

As mentioned above, DENV genotypes differ in virulence, including their human pathogenicities and epidemic potential. Therefore, phylogenetic studies were conducted to determine the epidemic potential of DENV isolates from Veracruz and Oaxaca. These studies were carried out using partial sequences of the genes encoding C-prM, NS5 
and NS3. C and prM were chosen because they represent a region that is commonly analyzed in studies of the molecular epidemiology of DEN $[17,26]$. NS5 and NS3 are important because they may be useful for determining the genotype and may help us to establish additional specific genetic markers. Also, NS5 has more nucleotide substitutions [27] and five of the eight amino acid differences between the American and Asian genotypes are located in the $\mathrm{N}$ terminus of this protein [12]. The RdRp of NS5 is crucial for viral RNA replication. NS5 also contains an MTase domain that is essential for the interaction with RdRp required for virus replication and/or infectivity [28]. In addition, NS5 has been used thoroughly in studies of flavivirus phylogenies [29,30].

The interdomain (linker) region 169-179 of NS3 and the two loops that encircle the entrance of the ATP binding pocket [31] were also studied because of the role of this protein in virus replication. This region has also been implicated in the intrinsic flexibility that couples movements between the NS3 protease and helicase domains [32].

In summary, phylogenetic and genetic analyses of Oaxaca and Veracuz virus isolates reveal the putative geographic origins of the viruses and potential molecular determinants of virulence.

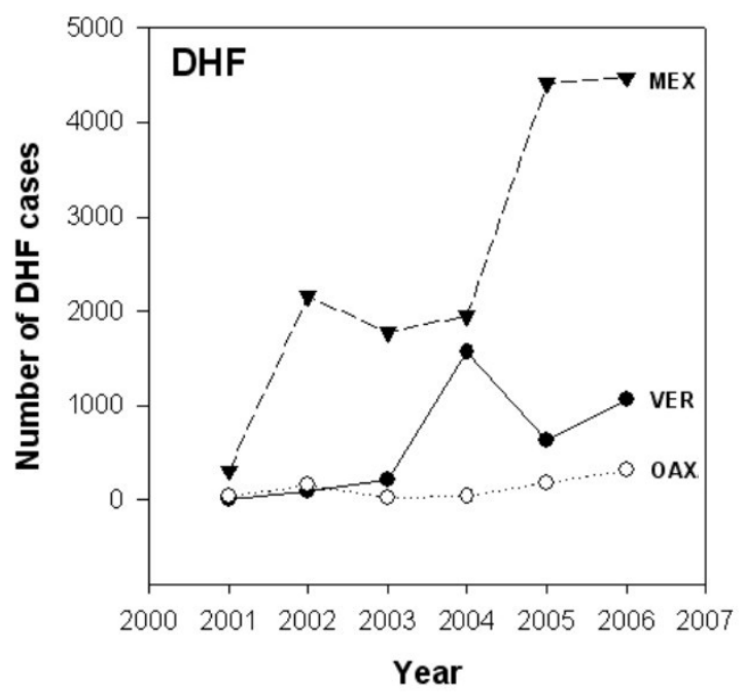

\section{Results}

\section{Virus isolation and serotyping}

The Mac-Elisa test has been implemented by the Ministry of Health in Mexico for diagnosing DENV infections [33]. Patients whose clinical reports show symptoms of DHF are exclusively evaluated using an IgG-ELISA kit. Reported cases are under-notified and the circulating serotype is not widely known. Analysis of the circulating serotype is important since it has been observed that DEN cases in Mexico are increasing annually (Figure 1). This Figure also shows that the percentage of DHF cases has increased in comparison to DF cases [34].

The serotypes of 88 DENV samples from different sites in Oaxaca and Veracruz States, Mexico (Figure 2) were evaluated and the results are shown in Figure 3. Serotyping was performed by RT-PCR using the RNA obtained from isolates in C6/36 cells; each test to determine serotype was repeated at least twice. C6/36 cells were chosen because the nucleotide sequence of the virus genome is conserved $[35,36]$.

Out of 27 isolates from Oaxaca, 18 were diagnosed with DHF; 12 were serotype 2 and six were serotype 1 (Table 1 ). Among the 61 isolates obtained from patient sera in Veracruz State, fifteen with DHF were serotype 2, two were serotype 1 and the isolate VER/MEX013A/06 showed both serotypes 1 and 2 .

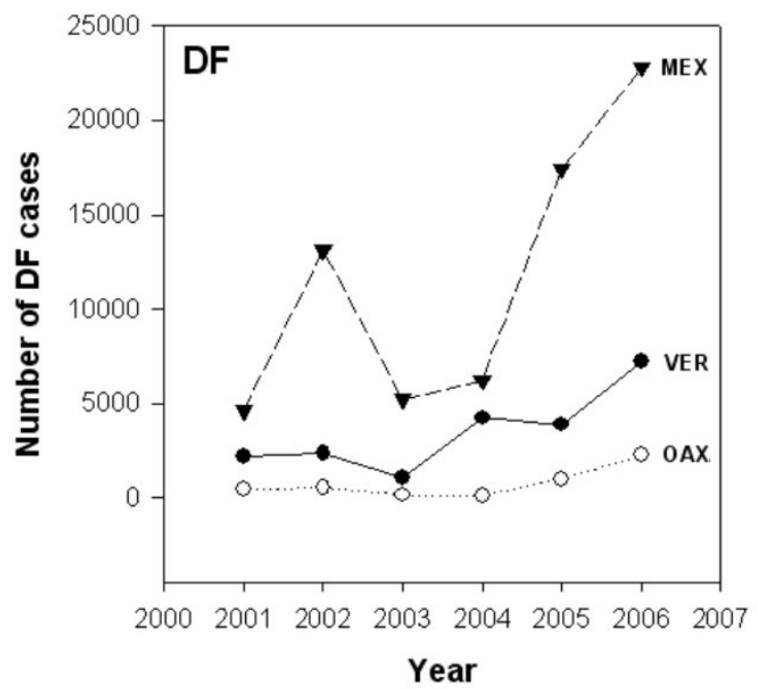

\section{Figure I}

Epidemiology of DEN in Mexico (2000-2006). The numbers of DF and DHF cases were obtained from CENAVECE [34]. The total numbers of DF and DHF cases in Oaxaca and Veracruz states are compared with the numbers in Mexico as a whole. 


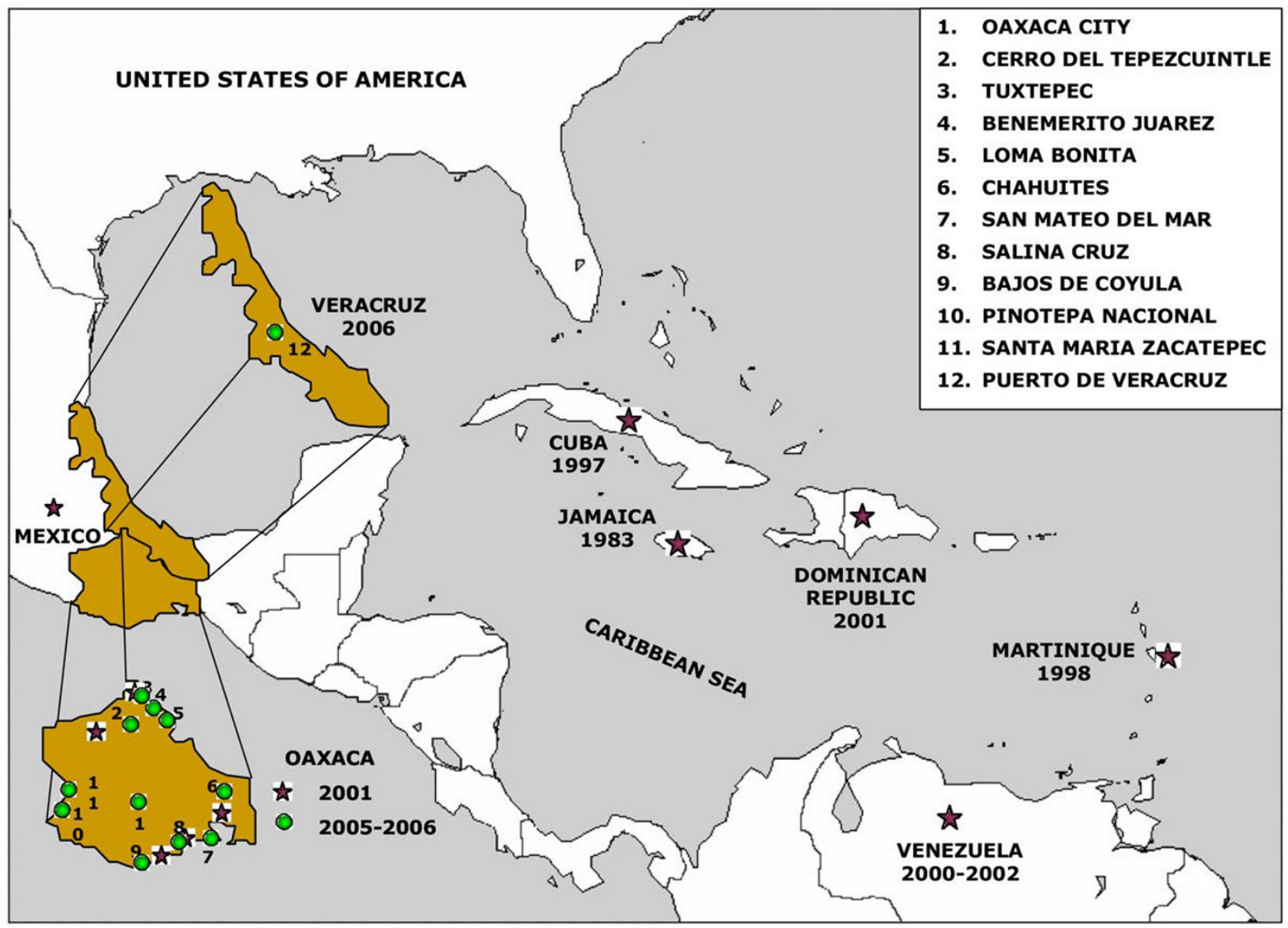

\section{Figure 2}

Mexican and Caribbean region map. The virus sample collection sites for the 13 isolates that were sequenced from Oaxaca and Veracruz States are shown in addition to the sites of origin of the GenBank sequences from the strains used in this phylogenetic study. These strains are from Caribbean countries and are grouped in the same branch as the sequences of our isolates corresponding to the Asian/American genotype.

Out of a total of 52 isolates with DF, 35 were serotype 2 seven serotype 3 , eight serotype 1 and two serotype 4 (Table 1). Known strains of DENV-1, $-2,-3$ and -4 were used as positive controls for the RT-PCR assays.

Figure 3 shows the percentages of cases of DENV-1 (22\%, $16 \%)$, DENV-2 (67\%, 72\%) and DENV-3 $(11 \%, 6 \%)$ in Oaxaca and Veracruz respectively; DENV-4 cases had the lowest percentages $(0 \%, 3 \%)$. These values were consistent in both States.

\section{Phylogenetic analysis of Oaxaca isolates}

Portions of the $\mathrm{C}_{91}-\mathrm{prM}_{1}, \mathrm{NS}_{73}, \mathrm{NS}_{2206}$ and $\mathrm{NS}_{484}$ genes were amplified and sequenced as described in the Methods section. Fourteen sequences were obtained from 8 isolates from Oaxaca and 6 from Veracruz for the C-prM fragment (11 with DF and 3 with DHF); 7 sequences were obtained from 5 Oaxaca isolates and 2 from Veracruz isolates for the MTase domain (NS5 $5_{73}$ ) fragment (4 with DF and 3 with DHF); 18 sequences were obtained from 7 Oaxaca isolates and 11 from Veracruz isolates for the RdRp domain (NS5 73 ) fragment (12 with DF and 6 with DHF); 7 sequences were obtained from 7 Oaxaca isolates for the helicase domain fragment (6 with DF and 1 with DHF).

Phylogenetic analysis was then performed on these sequences, including other prototype sequences of characterized isolates for DENV-2 (Table 2, 3).

This analysis showed that $\mathrm{C}_{91}-\mathrm{prM}_{1}, \mathrm{NS}_{75}$ and $\mathrm{NS}_{484}$ in the DENV-2 isolates from Oaxaca and Veracruz were most 


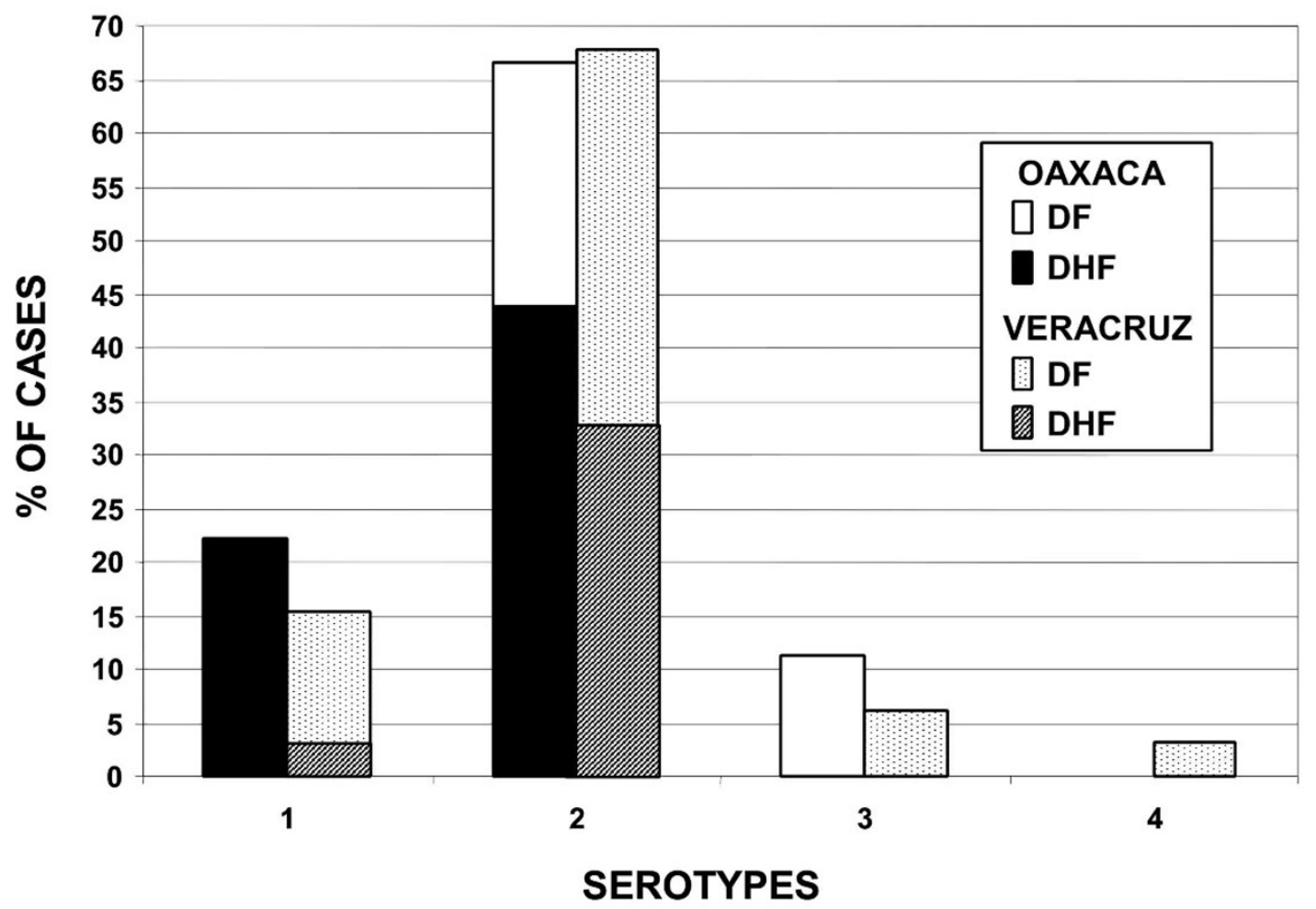

Figure 3

Summary of all DENV analyzed in this study. The percentages of DENV-I, 2, 3 and 4 detected in Oaxaca and Veracruz States during outbreaks in 2005-06. The graph shows that the relative percentages of DENV-I, 2, 3 and 4 do not differ between Oaxaca and Veracruz, although the numbers of samples analyzed were different.

closely related to strains from the Caribbean islands Cuba, Dominican Republic and Martinique (Figure 4). The phylogenetic tree for $\mathrm{NS}_{75}$ and $\mathrm{NS}_{484}$ is not shown. The strains from Brazil, Venezuela and Jamaica formed an independent clade. Interestingly, analysis of $\mathrm{C}_{91}-\mathrm{prM}_{1}$ showed that the isolates obtained in 2001 belonged to a

Table I: Serotype determination of isolates from Oaxaca (20052006) and Veracruz (2006).

\begin{tabular}{llll}
\hline SEROTYPE & DIAGNOSIS & OAXACA & VERACRUZ \\
\hline $\mathbf{I}$ & DHF & $\mathbf{6}$ & $\mathbf{2}$ \\
& DF & $\mathbf{0}$ & $\mathbf{8}$ \\
$\mathbf{2}$ & DHF & $\mathbf{1 2}$ & $\mathbf{1 5}$ \\
& DF & $\mathbf{6}$ & $\mathbf{2 9}$ \\
$\mathbf{3}$ & DF & $\mathbf{3}$ & $\mathbf{4}$ \\
$\mathbf{4}$ & DF & 0 & $\mathbf{2}$ \\
$\mathbf{1 , 2}$ & DHF & 0 & $\mathbf{1}$
\end{tabular}

different clade from those obtained in 2005-2006, with a high bootstrap support of $94 \%$.

Phylogenetic analysis of NS5 2206 gave a somewhat different pattern: essentially, the isolates belonged to the same clade as those from Venezuela, Brazil and Jamaica and were distinct from the Caribbean islands isolates (Figure 5). Bootstrap support was maintained in this analysis.

All the genotypes clustered in separate branches with high bootstrap support, as previously shown in phylogenetic analysis of other virus sequences $[25,17,37]$. Our analysis supports the view that the Asian/American strains are phylogenetically different from the main Asian and American genotypes.

\section{Sequence analysis of Oaxaca isolates}

Most of the amino acid (aa) differences were associated with nucleotide changes in the prM fragment $(13.6 \%, 6 /$ 
Table 2: Summary of consistent amino acid change among the five DENV-2 genotypes viruses.

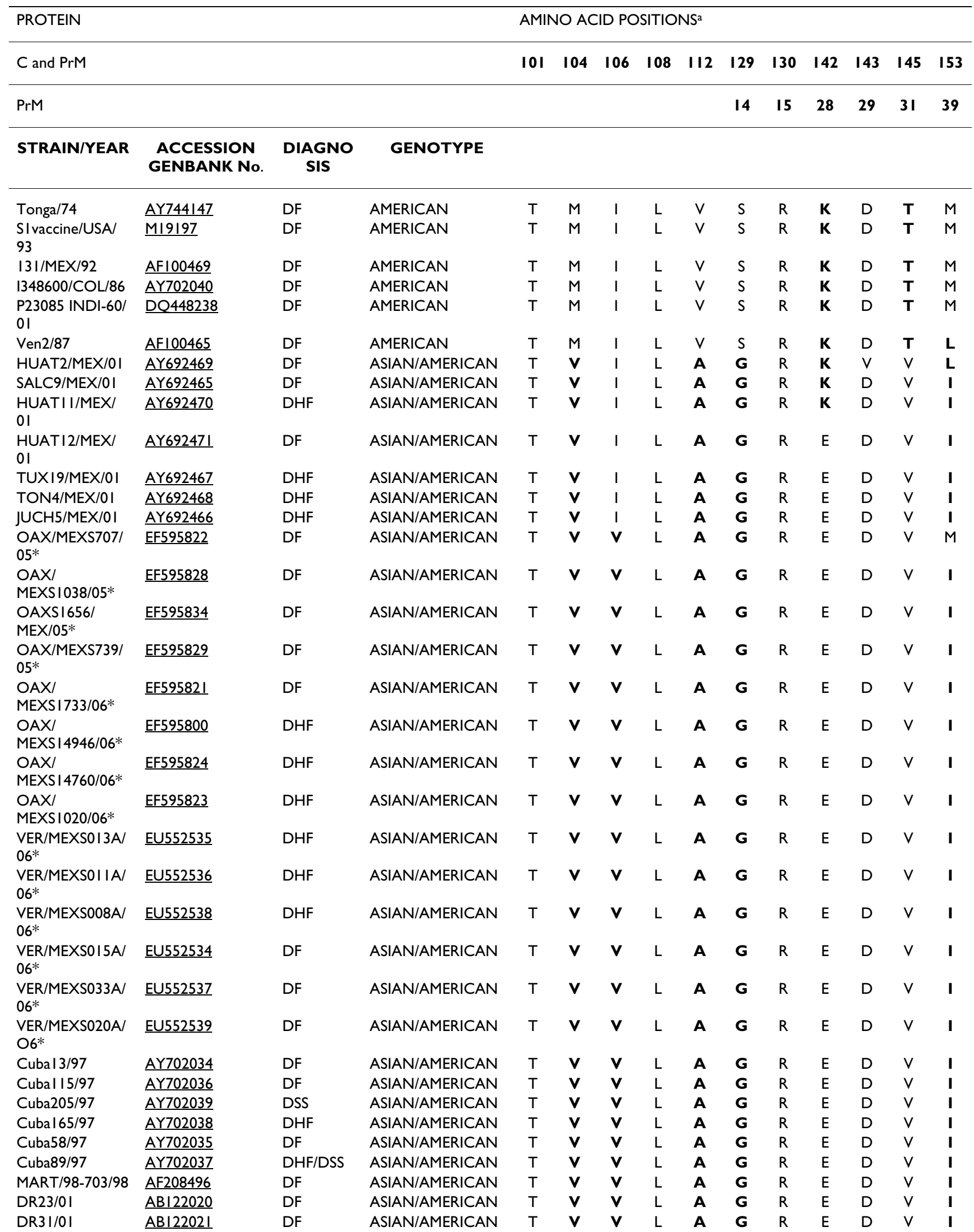


Table 2: Summary of consistent amino acid change among the five DENV-2 genotypes viruses. (Continued)

\begin{tabular}{|c|c|c|c|c|c|c|c|c|c|c|c|c|c|c|}
\hline DR59/0I & $\mathrm{AB} \mid 22022$ & DHF & ASIAN/AMERICAN & $T$ & $\mathbf{V}$ & $\mathbf{V}$ & $\mathrm{L}$ & $\mathbf{A}$ & $\mathbf{G}$ & $R$ & $E$ & $\mathrm{D}$ & $\mathrm{V}$ & $T$ \\
\hline $\begin{array}{l}\text { LARDI } 70 \text { I/Ven/ } \\
01\end{array}$ & AF36086I & DF & ASIAN/AMERICAN & $\mathrm{T}$ & $\mathbf{V}$ & $\mathbf{v}$ & $\mathrm{L}$ & $\mathbf{A}$ & G & $\mathrm{R}$ & $E$ & $\mathrm{D}$ & V & I \\
\hline $\begin{array}{l}\text { LARDI } 432 / \mathrm{Ven} / \\
01\end{array}$ & $\underline{A F 360860}$ & DF & ASIAN/AMERICAN & $\mathrm{T}$ & $\mathbf{v}$ & $\mathbf{v}$ & $\mathrm{L}$ & $\mathbf{A}$ & G & $\mathrm{R}$ & $E$ & $\mathrm{D}$ & V & $\mathbf{I}$ \\
\hline $\begin{array}{l}\text { LARDI9I0/Ven/ } \\
01\end{array}$ & $\underline{A F 360862}$ & DF & ASIAN/AMERICAN & $\mathrm{T}$ & $\mathbf{V}$ & I & $\mathrm{L}$ & $\mathbf{A}$ & G & $\mathrm{R}$ & $E$ & $\mathrm{D}$ & V & I \\
\hline $\begin{array}{l}\text { LARDI996/Ven/ } \\
\text { 01 }\end{array}$ & $\underline{A F 360863}$ & DF & ASIAN/AMERICAN & $\mathrm{T}$ & $\mathbf{V}$ & I & $\mathrm{L}$ & $\mathbf{A}$ & G & $\mathrm{R}$ & $E$ & $\mathrm{D}$ & V & $\mathbf{I}$ \\
\hline Mara3/Ven/02 & AY0444421 & DHF & ASIAN/AMERICAN & $\mathrm{T}$ & $\mathbf{V}$ & I & L & $\mathbf{A}$ & G & $\mathrm{R}$ & $E$ & $\mathrm{D}$ & V & $\mathbf{I}$ \\
\hline BR64022/02 & AF489932 & NA & ASIAN/AMERICAN & $\mathrm{T}$ & $\mathbf{v}$ & I & L & $\mathbf{A}$ & G & $\mathrm{R}$ & $E$ & $\mathrm{D}$ & V & $\mathbf{I}$ \\
\hline Jamaica//409/83 & M20558 & DHF & ASIAN/AMERICAN & $\mathrm{T}$ & $\mathbf{V}$ & I & $\mathrm{L}$ & $\mathbf{A}$ & G & $\mathrm{R}$ & $E$ & $\mathrm{D}$ & V & $\mathbf{I}$ \\
\hline China 04 & $\underline{\text { U8741II }}$ & DHF & ASIAN/AMERICAN & $\mathrm{T}$ & $\mathbf{V}$ & I & L & $\mathbf{A}$ & G & $\mathrm{R}$ & E & $\mathrm{D}$ & V & $\mathbf{I}$ \\
\hline CO390/Th/99 & AFI00462 & DHF & ASIAN I & $\mathbf{S}$ & $\mathbf{V}$ & I & L & V & $S$ & $\mathrm{R}$ & $\mathrm{E}$ & $\mathrm{D}$ & V & M \\
\hline Bangkok 1974 & A)48727I & NA & ASIAN I & $\mathbf{S}$ & $M$ & I & $\mathrm{L}$ & V & $S$ & $\mathrm{R}$ & $E$ & $\mathrm{D}$ & V & M \\
\hline I668I/USA/97 & $\underline{\text { U874III }}$ & NA & ASIAN I & $\mathbf{s}$ & M & I & $\mathrm{L}$ & V & $S$ & $\mathrm{R}$ & E & V & V & M \\
\hline PDK53/USA/97 & $\underline{\mathrm{U} 87412}$ & NA & ASIAN I & $\mathbf{S}$ & $M$ & I & $\mathrm{L}$ & v & $S$ & $\mathrm{R}$ & $E$ & V & V & M \\
\hline 1668I-PDK53/97 & $\underline{\text { U8741II }}$ & DHF & ASIAN I & $\mathbf{S}$ & $M$ & I & L & V & $S$ & $\mathrm{R}$ & E & $\mathrm{D}$ & V & M \\
\hline ThNH-p I I/93 & AF022437 & DF & ASIAN I & $\mathbf{S}$ & $M$ & I & L & V & $S$ & $\mathrm{R}$ & $E$ & $\mathrm{D}$ & V & M \\
\hline ThNH-pl2/93 & AF022438 & DF & ASIAN I & $\mathbf{S}$ & $M$ & I & $\mathrm{L}$ & V & $S$ & $\mathrm{R}$ & $E$ & $\mathrm{D}$ & V & M \\
\hline ThNH-p I 4/93 & AF022439 & DHF & ASIAN I & $\mathbf{S}$ & $M$ & I & L & V & $S$ & $\mathrm{R}$ & $E$ & D & V & M \\
\hline ThNH-7/93 & AF022434 & DSS & ASIAN I & $\mathbf{S}$ & $M$ & I & L & V & $S$ & I & $E$ & $\mathrm{D}$ & V & M \\
\hline ThNH-28/93 & $\overline{\mathrm{AF} 022435}$ & DHF & ASIAN I & $\mathbf{S}$ & $M$ & I & $\mathrm{L}$ & V & $S$ & $\mathbf{I}$ & $E$ & $\mathrm{D}$ & V & M \\
\hline ThNH-52/93 & AF022436 & DHF & ASIAN I & $\mathbf{S}$ & $M$ & I & L & V & $S$ & I & E & D & V & M \\
\hline ThNH-pl6/93 & AF022440 & DF & ASIAN I & $\mathbf{S}$ & $M$ & I & L & V & $S$ & I & $E$ & D & V & M \\
\hline ThNH-p36/93 & AF022441 & DHF & ASIAN I & $\mathbf{S}$ & $M$ & I & $\mathrm{L}$ & V & $S$ & $\mathbf{I}$ & $E$ & D & V & M \\
\hline ThNH29/93 & AFI69678 & DHF & ASIAN I & $\mathbf{S}$ & $M$ & I & L & V & $S$ & I & $E$ & $\mathrm{D}$ & V & M \\
\hline $\mathrm{ThNH} 36 / 93$ & AFI69679 & DF & ASIAN I & $\mathbf{S}$ & $M$ & I & L & V & $S$ & I & $E$ & $D$ & V & M \\
\hline ThNH45/93 & AFI69680 & DHF & ASIAN I & $\mathbf{S}$ & $M$ & I & L & V & $S$ & I & $E$ & $D$ & V & M \\
\hline ThNH55/93 & AFI6968I & DHF & ASIAN I & $\mathbf{S}$ & $M$ & I & L & V & $S$ & I & E & D & V & M \\
\hline ThNH54/93 & AFI69682 & DHF & ASIAN I & $\mathbf{S}$ & $M$ & I & $\mathrm{L}$ & v & $S$ & $\mathbf{I}$ & $E$ & D & V & M \\
\hline ThNH62/93 & AFI69683 & DHF & ASIAN I & $\mathbf{S}$ & M & I & L & V & $S$ & I & E & D & V & M \\
\hline $\mathrm{ThNH} 63 / 93$ & AFI69684 & DHF & ASIAN I & $\mathbf{S}$ & $M$ & I & L & V & $S$ & I & $E$ & $D$ & V & M \\
\hline ThNH69/93 & AFI69685 & DHF & ASIAN I & $\mathbf{S}$ & $M$ & I & $\mathrm{L}$ & v & $S$ & $\mathbf{I}$ & E & $\mathrm{D}$ & V & M \\
\hline ThNH73/93 & AFI69686 & DHF & ASIAN I & $\mathbf{S}$ & M & I & L & V & $S$ & I & $E$ & D & V & M \\
\hline ThNH76/93 & AFI69687 & DHF & ASIAN I & $\mathbf{S}$ & $M$ & I & L & V & $S$ & I & $E$ & $D$ & V & M \\
\hline ThNH8I/93 & AFI69688 & DHF & ASIAN I & $\mathbf{S}$ & $M$ & I & $\mathrm{L}$ & v & $S$ & $\mathbf{I}$ & $E$ & D & V & M \\
\hline Mara $4 / \mathrm{Ven} / 90^{\&}$ & $\underline{A F I 00466}$ & DHF & ASIAN I & $\mathbf{S}$ & $M$ & I & L & V & $S$ & I & E & D & V & M \\
\hline K0008/Th/99 & AFI00459 & DHF & ASIAN I & $\mathbf{S}$ & $M$ & I & L & v & $S$ & $\mathbf{I}$ & $E$ & $\mathrm{D}$ & V & M \\
\hline $\mathrm{K} 0010 / \mathrm{Th} / 99$ & $\underline{\mathrm{AFI} 100460}$ & DF & ASIAN I & $\mathbf{s}$ & M & I & $\mathrm{L}$ & V & $S$ & I & $E$ & D & V & M \\
\hline C037I/Th/99 & $\underline{\mathrm{AFI} 100461}$ & DF & ASIAN I & $\mathbf{S}$ & $M$ & I & L & V & $S$ & I & E & D & V & M \\
\hline $\mathrm{C0166/Th/99}$ & $\overline{\mathrm{AFI} 100463}$ & DF & ASIAN I & $\mathbf{S}$ & $M$ & I & L & v & $S$ & $\mathbf{I}$ & $E$ & $\mathrm{D}$ & V & M \\
\hline $\mathrm{C0167/Th/99}$ & $\underline{\mathrm{AFI}} 00464$ & DHF & ASIAN I & $\mathbf{s}$ & M & I & $\mathrm{L}$ & V & $S$ & I & $E$ & D & V & M \\
\hline $\begin{array}{l}\text { New Guinea C/ } \\
44\end{array}$ & D00346 & DHF & ASIAN 2 & $\mathrm{~T}$ & M & I & L & V & $S$ & $\mathrm{R}$ & E & D & V & M \\
\hline 43/China/89 & $\underline{A F 204178}$ & DF & ASIAN 2 & $\mathrm{~T}$ & $M$ & I & L & V & $S$ & $\mathrm{R}$ & G & $\mathrm{D}$ & V & M \\
\hline 44/China/87 & $\underline{A F 204177}$ & DF & ASIAN 2 & $\mathrm{~T}$ & M & I & $\mathrm{L}$ & V & $S$ & $\mathrm{R}$ & G & D & V & M \\
\hline BA05i/Indon/05 & AY858035 & DF & COSMOPOLITAN & $\mathrm{T}$ & I & I & $\mathbf{M}$ & V & $S$ & $\mathrm{R}$ & $E$ & $\mathbf{N}$ & V & M \\
\hline TSV0I/Austra/93 & AY037116 & NA & COSMOPOLITAN & $\mathrm{T}$ & I & I & $\mathbf{M}$ & v & $S$ & $\mathrm{R}$ & $E$ & $\mathbf{N}$ & V & M \\
\hline $\begin{array}{l}98900666 / \text { Indo/ } \\
04\end{array}$ & $\mathrm{AB} 189124$ & DSS & COSMOPOLITAN & $\mathrm{T}$ & $\mathbf{v}$ & I & $\mathbf{M}$ & V & $S$ & $\mathrm{R}$ & $\mathrm{E}$ & $\mathbf{N}$ & V & M \\
\hline ZSOI/China/OI & $\underline{E F 051521}$ & NA & COSMOPOLITAN & $\mathrm{T}$ & V & 1 & $\mathbf{M}$ & $\mathrm{V}$ & $\mathrm{s}$ & $\mathrm{R}$ & $E$ & $\mathbf{N}$ & V & M \\
\hline GWLI8/INDI/0I & DQ448231 & NA & COSMOPOLITAN & $\mathrm{T}$ & $\mathbf{v}$ & I & $\mathrm{L}$ & $\mathbf{A}$ & $S$ & $\mathrm{R}$ & $E$ & D & V & M \\
\hline GWL39/INDI/0I & DQ448232 & NA & COSMOPOLITAN & $\mathrm{T}$ & $\mathbf{V}$ & I & $\mathrm{L}$ & $\mathbf{A}$ & $S$ & $\mathrm{R}$ & $E$ & $D$ & V & M \\
\hline
\end{tabular}

aAmino acid sequence was numbered starting from protein $C$ or prM to the end of the last codon sequenced. *Sequences of this study.

\&Recombinant strain [16]. 
Table 3: Summary of consistent amino acid change among the five DENV-2 genotype viruses.

\begin{tabular}{|c|c|c|c|c|c|c|c|c|c|c|c|c|c|c|c|c|c|c|}
\hline \multirow{3}{*}{$\begin{array}{l}\text { PROTEIN } \\
\\
\text { NS5 }\end{array}$} & & & \multirow{3}{*}{ DOMAIN } & \multicolumn{15}{|c|}{ AMINO ACID POSITIONSA } \\
\hline & & & & \multicolumn{4}{|c|}{ MTase } & \multicolumn{8}{|c|}{ RdRp } & \multicolumn{3}{|c|}{ Helicase $†$} \\
\hline & & & & 026 & 155 & 179 & 192 & 748 & 762 & 784 & 799 & 810 & 818 & 835 & 864 & & & \\
\hline NS3 & & & & & & & & & & & & & & & & 187 & 249 & 250 \\
\hline $\begin{array}{l}\text { STRAIN/ } \\
\text { YEAR }\end{array}$ & $\begin{array}{l}\text { ACCESSION } \\
\text { GENBANK }\end{array}$ & DIAGNOSIS & GENOTYPE & & & & & & & & & & & & & & & \\
\hline Tonga/74 & AY744I47 & DF & AMERICAN & 1 & $\vee$ & $\mathbf{v}$ & $\mathbf{R}$ & $\mathrm{R}$ & $S$ & $S$ & $\mathbf{S}$ & $\mathrm{T}$ & $\mathbf{L}$ & 1 & $\mathrm{~T}$ & $\mathrm{R}$ & $\mathrm{R}$ & A \\
\hline $\begin{array}{l}\text { SI vaccine/USA/ } \\
93\end{array}$ & $\underline{M 19197}$ & NA & AMERICAN & 1 & V & $\mathbf{v}$ & $\mathbf{R}$ & $\mathrm{R}$ & $\mathrm{S}$ & $S$ & $\mathbf{S}$ & $\mathrm{T}$ & $\mathbf{L}$ & 1 & $\mathrm{~T}$ & $\mathrm{R}$ & $\mathrm{R}$ & A \\
\hline $131 / M E X / 92$ & AFI00469 & DF & AMERICAN & 1 & $\vee$ & $\mathbf{v}$ & $\mathbf{R}$ & $\mathrm{R}$ & $S$ & $\mathrm{~S}$ & $\mathbf{S}$ & $\mathrm{T}$ & $\mathbf{L}$ & $\mathrm{I}$ & $\mathrm{T}$ & $\mathrm{R}$ & $\mathrm{R}$ & A \\
\hline Ven2/87 & AFI00465 & DF & AMERICAN & 1 & V & $\mathbf{v}$ & $\mathbf{R}$ & $\mathrm{R}$ & $\mathrm{S}$ & $S$ & $\mathbf{S}$ & $\mathrm{T}$ & $\mathbf{L}$ & 1 & $\mathrm{~T}$ & $\mathrm{R}$ & $\mathrm{R}$ & A \\
\hline 1348600/COL/86 & AY702040 & DF & AMERICAN & 1 & V & $\mathbf{v}$ & $\mathbf{R}$ & $\mathrm{R}$ & $\mathrm{S}$ & $\mathrm{S}$ & $\mathbf{S}$ & $\mathrm{T}$ & $\mathbf{L}$ & 1 & $\mathrm{~T}$ & $\mathrm{R}$ & $\mathrm{R}$ & A \\
\hline $\begin{array}{l}\text { OAX/MEX/ } \\
\text { S707/05§ }\end{array}$ & EF595821 & DF & $\begin{array}{l}\text { ASIAN/ } \\
\text { AMERICAN }\end{array}$ & - & - & - & - & $\mathbf{K}$ & $\mathbf{T}$ & $\mathbf{P}$ & $\mathrm{K}$ & A & Q & $\mathbf{v}$ & $\mathrm{T}$ & $\mathbf{K}$ & $\mathbf{K}$ & $\mathbf{T}$ \\
\hline $\begin{array}{l}\text { OAXI } \\
\text { MEXSI038/05§ }\end{array}$ & $\begin{array}{l}\text { EF595817/ } \\
\underline{E F 595825}\end{array}$ & DF & $\begin{array}{l}\text { ASIAN/ } \\
\text { AMERICAN }\end{array}$ & $\mathbf{v}$ & I & 1 & $\mathrm{~K}$ & $\mathbf{K}$ & $\mathbf{T}$ & $\mathbf{P}$ & $\mathrm{K}$ & A & Q & $\mathbf{v}$ & $\mathrm{T}$ & $\mathbf{K}$ & $\mathbf{K}$ & $\mathbf{T}$ \\
\hline $\begin{array}{l}\text { OAX/ } \\
\text { MEXSI656/05§ }\end{array}$ & $\begin{array}{l}\text { EF595815/ } \\
\text { EF595820 }\end{array}$ & DF & $\begin{array}{l}\text { ASIAN/ } \\
\text { AMERICAN }\end{array}$ & $\mathbf{v}$ & I & 1 & $\mathrm{~K}$ & $\mathbf{K}$ & $\mathbf{T}$ & $\mathbf{P}$ & $\mathrm{K}$ & $\mathbf{A}$ & Q & $\mathbf{v}$ & $\mathrm{T}$ & $\mathbf{K}$ & $\mathbf{K}$ & $\mathbf{T}$ \\
\hline $\begin{array}{l}\text { OAX/MEXS739/ } \\
05 \S\end{array}$ & $\begin{array}{l}\text { EF595818/ } \\
\text { EF595822 }\end{array}$ & DF & $\begin{array}{l}\text { ASIAN/ } \\
\text { AMERICAN }\end{array}$ & $\mathbf{v}$ & $\mathbf{I}$ & 1 & $\mathrm{~K}$ & $\mathbf{K}$ & $\mathbf{T}$ & $\mathbf{P}$ & $\mathrm{K}$ & A & Q & $\mathbf{v}$ & $\mathrm{T}$ & $\mathbf{K}$ & $\mathbf{K}$ & $\mathbf{T}$ \\
\hline $\begin{array}{l}\text { OAXI } \\
\text { MEXSI020/06\$ }\end{array}$ & $\begin{array}{l}\text { EF595816/ } \\
\text { EF595824 }\end{array}$ & DHF & $\begin{array}{l}\text { ASIAN/ } \\
\text { AMERICAN }\end{array}$ & $\mathbf{v}$ & I & 1 & $\mathrm{~K}$ & $\mathbf{K}$ & $\mathbf{T}$ & $\mathbf{P}$ & $\mathrm{K}$ & A & Q & $\mathbf{v}$ & $\mathrm{T}$ & $\mathbf{K}$ & $\mathbf{K}$ & $\mathbf{T}$ \\
\hline $\begin{array}{l}\text { OAXI } \\
\text { MEXSI733/06\$ }\end{array}$ & $\begin{array}{l}\text { EF595819/ } \\
\text { EF595823 }\end{array}$ & DF & $\begin{array}{l}\text { ASIAN/ } \\
\text { AMERICAN }\end{array}$ & $\mathbf{v}$ & I & 1 & $\mathrm{~K}$ & $\mathbf{K}$ & $\mathbf{T}$ & $\mathbf{P}$ & $\mathrm{K}$ & A & Q & $\mathbf{v}$ & $\mathrm{T}$ & $\mathbf{K}$ & $\mathbf{K}$ & $\mathbf{T}$ \\
\hline $\begin{array}{l}\text { VER/MEX024A/ } \\
06 \S\end{array}$ & $\begin{array}{l}\text { EU570975/ } \\
\text { EU570983 }\end{array}$ & $\mathrm{DHF}$ & $\begin{array}{l}\text { ASIAN/ } \\
\text { AMERICAN }\end{array}$ & $\mathbf{v}$ & I & 1 & $\mathrm{~K}$ & $\mathbf{K}$ & $\mathbf{T}$ & $\mathbf{P}$ & $\mathrm{K}$ & A & Q & $\mathbf{v}$ & $\mathrm{T}$ & - & - & - \\
\hline $\begin{array}{l}\text { VER/MEX0I3A/ } \\
06 \S\end{array}$ & $\begin{array}{l}\text { EU570980/ } \\
\text { EU570982 }\end{array}$ & DHF & $\begin{array}{l}\text { ASIAN/ } \\
\text { AMERICAN }\end{array}$ & $\mathbf{v}$ & I & 1 & $\mathrm{~K}$ & $\mathbf{K}$ & $\mathbf{T}$ & $\mathbf{P}$ & $\mathrm{K}$ & A & Q & $\mathbf{v}$ & $\mathrm{T}$ & - & - & - \\
\hline Cuba I $3 / 97$ & AY702034 & DF & $\begin{array}{l}\text { ASIAN/ } \\
\text { AMERICAN }\end{array}$ & 1 & I & 1 & $\mathrm{~K}$ & $\mathbf{K}$ & $\mathbf{T}$ & $S$ & $\mathrm{~K}$ & A & $\mathrm{Q}$ & $\mathbf{v}$ & $\mathrm{T}$ & $\mathbf{K}$ & $\mathrm{R}$ & A \\
\hline CubalI5/97 & AY702036 & DF & $\begin{array}{l}\text { ASIAN/ } \\
\text { AMERICAN }\end{array}$ & 1 & I & 1 & $\mathrm{~K}$ & $\mathbf{K}$ & $\mathbf{T}$ & $S$ & $\mathrm{~K}$ & A & $\mathrm{Q}$ & $\mathbf{v}$ & $\mathrm{T}$ & K & $\mathrm{R}$ & A \\
\hline Cuba205/97 & AY702039 & DSS & $\begin{array}{l}\text { ASIAN/ } \\
\text { AMERICAN }\end{array}$ & 1 & I & 1 & $\mathrm{~K}$ & $\mathbf{K}$ & $\mathbf{T}$ & $S$ & $\mathrm{~K}$ & A & $\mathrm{Q}$ & $\mathbf{v}$ & $\mathrm{T}$ & $\mathbf{K}$ & $\mathrm{R}$ & A \\
\hline Cuba 165/97 & $\underline{\text { AY702038 }}$ & $\mathrm{DHF}$ & $\begin{array}{l}\text { ASIAN/ } \\
\text { AMERICAN }\end{array}$ & 1 & I & 1 & $\mathrm{~K}$ & $\mathbf{K}$ & $\mathbf{T}$ & $S$ & $\mathrm{~K}$ & A & Q & $\mathbf{v}$ & $\mathrm{T}$ & $\mathbf{K}$ & $\mathrm{R}$ & A \\
\hline Cuba58/97 & AY702035 & DF & $\begin{array}{l}\text { ASIAN/ } \\
\text { AMERICAN }\end{array}$ & 1 & I & 1 & $\mathrm{~K}$ & $\mathbf{K}$ & $\mathbf{T}$ & $\mathrm{S}$ & $\mathrm{K}$ & A & Q & $\mathbf{v}$ & $\mathrm{T}$ & $\mathbf{K}$ & $\mathrm{R}$ & A \\
\hline Cuba89/97 & AY702037 & DHF/DSS & $\begin{array}{l}\text { ASIAN/ } \\
\text { AMERICAN }\end{array}$ & 1 & I & 1 & $\mathrm{~K}$ & $\mathbf{K}$ & $\mathbf{T}$ & $S$ & $\mathrm{~K}$ & A & $\mathrm{Q}$ & $\mathbf{v}$ & $\mathrm{T}$ & $\mathbf{K}$ & $\mathrm{R}$ & A \\
\hline $\begin{array}{l}\text { MART/98-703/ } \\
98\end{array}$ & AF208496 & DF & $\begin{array}{l}\text { ASIAN/ } \\
\text { AMERICAN }\end{array}$ & 1 & $\mathbf{I}$ & 1 & $\mathrm{~K}$ & $\mathbf{K}$ & $\mathbf{T}$ & $\mathrm{S}$ & $\mathrm{K}$ & A & Q & $\mathbf{v}$ & $\mathrm{T}$ & K & $\mathrm{R}$ & A \\
\hline DR23/0I & $A B \mid 22020$ & DF & $\begin{array}{l}\text { ASIAN/ } \\
\text { AMERICAN }\end{array}$ & 1 & $\mathbf{I}$ & 1 & $\mathrm{~K}$ & $\mathbf{K}$ & $\mathbf{T}$ & $S$ & $\mathrm{~K}$ & $\mathbf{A}$ & Q & $\mathbf{v}$ & $\mathrm{T}$ & $\mathbf{K}$ & $\mathrm{R}$ & A \\
\hline DR3I/0I & $\underline{A B \mid 22021}$ & DF & $\begin{array}{l}\text { ASIAN/ } \\
\text { AMERICAN }\end{array}$ & 1 & I & 1 & $\mathrm{~K}$ & $\mathbf{K}$ & $\mathbf{T}$ & $S$ & K & A & Q & $\mathbf{v}$ & $\mathrm{T}$ & $\mathbf{K}$ & $\mathrm{R}$ & A \\
\hline DR59/0I & $\underline{A B \mid 22022}$ & $\mathrm{DHF}$ & $\begin{array}{l}\text { ASIAN/ } \\
\text { AMERICAN }\end{array}$ & 1 & $\mathbf{I}$ & 1 & $\mathrm{~K}$ & $\mathbf{K}$ & $\mathbf{T}$ & $S$ & $\mathrm{~K}$ & A & $\mathrm{Q}$ & $\mathbf{v}$ & $\mathrm{T}$ & $\mathbf{K}$ & $\mathrm{R}$ & A \\
\hline BR64022/02 & AF489932 & NA & $\begin{array}{l}\text { ASIAN/ } \\
\text { AMERICAN }\end{array}$ & 1 & I & 1 & $\mathrm{~K}$ & $\mathbf{K}$ & $\mathbf{T}$ & $S$ & K & A & $\mathrm{Q}$ & $\mathbf{v}$ & $\mathrm{T}$ & $\mathbf{K}$ & $\mathrm{R}$ & A \\
\hline Mara $4 \& / V e n / 90$ & AFI00466 & $\mathrm{DHF}$ & $\begin{array}{l}\text { ASIAN/ } \\
\text { AMERICAN }\end{array}$ & 1 & I & 1 & $\mathrm{~K}$ & $\mathbf{K}$ & $\mathbf{T}$ & $\mathrm{S}$ & $\mathrm{K}$ & A & Q & 1 & $\mathrm{~T}$ & $\mathbf{K}$ & $\mathrm{R}$ & A \\
\hline $\begin{array}{l}\text { Jamaica/N.I409/ } \\
83\end{array}$ & $\underline{M 20558}$ & NA & $\begin{array}{l}\text { ASIAN/ } \\
\text { AMERICAN }\end{array}$ & 1 & $\mathbf{I}$ & 1 & $\mathrm{~K}$ & $\mathbf{K}$ & $\mathbf{T}$ & $S$ & $\mathrm{~K}$ & A & $\mathrm{Q}$ & $\mathbf{v}$ & $\mathrm{T}$ & $\mathbf{K}$ & $\mathrm{R}$ & A \\
\hline China 04/85 & AFII966I & $\mathrm{DHF}$ & $\begin{array}{l}\text { ASIAN/ } \\
\text { AMERICAN }\end{array}$ & 1 & $\mathbf{I}$ & 1 & $\mathrm{~K}$ & $\mathbf{K}$ & $\mathbf{S}$ & $S$ & $\mathrm{~K}$ & $\mathrm{~T}$ & $\mathrm{Q}$ & $\mathbf{v}$ & $\mathrm{T}$ & $\mathbf{K}$ & $\mathrm{R}$ & $\mathbf{T}$ \\
\hline Bangkok 1974 & A)487271 & NA & ASIAN I & 1 & V & 1 & $\mathrm{~K}$ & $\mathrm{R}$ & $\mathbf{T}$ & $S$ & $\mathrm{~K}$ & $\mathrm{~T}$ & $\mathrm{Q}$ & 1 & $\mathbf{A}$ & $\mathrm{R}$ & $\mathrm{R}$ & A \\
\hline CO390/Th/99 & AFI00462 & $\mathrm{DHF}$ & ASIAN I & 1 & V & 1 & $\mathrm{~K}$ & $\mathrm{R}$ & $\mathrm{S}$ & $S$ & $\mathrm{~K}$ & $\mathrm{~T}$ & $\mathrm{Q}$ & 1 & $\mathbf{A}$ & $\mathrm{R}$ & $\mathrm{R}$ & A \\
\hline I668I/USA/97 & U8741I & NA & ASIAN I & 1 & V & 1 & $\mathrm{~K}$ & $\mathrm{R}$ & $\mathrm{S}$ & $S$ & $\mathrm{~K}$ & $\mathrm{~T}$ & $\mathrm{Q}$ & 1 & $\mathbf{A}$ & $\mathrm{R}$ & $\mathrm{R}$ & A \\
\hline PDK53/USA/97 & $\underline{\mathrm{U} 87412}$ & NA & ASIAN I & 1 & V & 1 & $\mathrm{~K}$ & $\mathrm{R}$ & $S$ & $S$ & $\mathrm{~K}$ & $\mathrm{~T}$ & Q & 1 & $\mathbf{A}$ & $\mathrm{R}$ & $\mathrm{R}$ & A \\
\hline $\begin{array}{l}\text { I668I-PDK53/ } \\
97\end{array}$ & $\overline{\mathrm{U} 8741 \mathrm{I}}$ & $\mathrm{DHF}$ & ASIAN I & 1 & $\vee$ & 1 & $\mathrm{~K}$ & $\mathrm{R}$ & $\mathrm{S}$ & $S$ & $\mathrm{~K}$ & $\mathrm{~T}$ & Q & 1 & A & $\mathrm{R}$ & $\mathrm{R}$ & $A$ \\
\hline ThNH-pl I/93 & AF022437 & DF & ASIAN I & 1 & $\vee$ & 1 & $\mathrm{~K}$ & $\mathrm{R}$ & $\mathrm{S}$ & $\mathrm{S}$ & $\mathrm{K}$ & $\mathrm{T}$ & $\mathrm{Q}$ & 1 & A & $\mathrm{R}$ & $\mathrm{R}$ & A \\
\hline ThNH-p I/93 & $\overline{\mathrm{AF} 022438}$ & DF & ASIAN I & 1 & $\vee$ & 1 & $\mathrm{~K}$ & $\mathrm{R}$ & $\mathrm{S}$ & $S$ & $\mathrm{~K}$ & $\mathrm{~T}$ & $\mathrm{Q}$ & 1 & A & $\mathrm{R}$ & $\mathrm{R}$ & $A$ \\
\hline ThNH-pl4/93 & AF022439 & DHF & ASIAN I & 1 & V & 1 & $\mathrm{~K}$ & $\mathrm{R}$ & $S$ & $S$ & $\mathrm{~K}$ & $\mathrm{~T}$ & $\mathrm{Q}$ & 1 & A & $\mathrm{R}$ & $\mathrm{R}$ & A \\
\hline ThNH-7/93 & AF022434 & DSS & ASIAN I & 1 & V & 1 & $\mathrm{~K}$ & $\mathrm{R}$ & $\mathrm{S}$ & $S$ & $\mathrm{~K}$ & $\mathrm{~T}$ & $\mathrm{Q}$ & 1 & A & $\mathrm{R}$ & $\mathrm{R}$ & A \\
\hline ThNH-28/93 & AF022435 & $\mathrm{DHF}$ & ASIAN I & 1 & $\vee$ & 1 & $\mathrm{~K}$ & $\mathrm{R}$ & $\mathrm{S}$ & $S$ & $\mathrm{~K}$ & $\mathrm{~T}$ & $\mathrm{Q}$ & 1 & $\mathbf{A}$ & $\mathrm{R}$ & $\mathrm{R}$ & A \\
\hline ThNH-52/93 & AF022436 & $\mathrm{DHF}$ & ASIAN I & 1 & $\vee$ & 1 & $\mathrm{~K}$ & $\mathrm{R}$ & $\mathrm{S}$ & $\mathrm{S}$ & $\mathrm{K}$ & $\mathrm{T}$ & $\mathrm{Q}$ & 1 & A & $\mathrm{R}$ & $\mathrm{R}$ & $A$ \\
\hline ThNH-p 16/93 & AF022440 & DF & ASIAN I & 1 & V & 1 & $\mathrm{~K}$ & $\mathrm{R}$ & $\mathrm{S}$ & $\mathrm{S}$ & $\mathrm{K}$ & $\mathrm{T}$ & $\mathrm{Q}$ & 1 & $\mathbf{A}$ & $\mathrm{R}$ & $\mathrm{R}$ & $A$ \\
\hline ThNH-p36/93 & AF022441 & $\mathrm{DHF}$ & ASIAN I & 1 & V & 1 & $\mathrm{~K}$ & $\mathrm{R}$ & $\mathrm{S}$ & $S$ & $\mathrm{~K}$ & $\mathrm{~T}$ & Q & 1 & $\mathbf{A}$ & $\mathrm{R}$ & $\mathrm{R}$ & A \\
\hline ThNH29/93 & AFI69678 & $\mathrm{DHF}$ & ASIAN I & 1 & V & 1 & $\mathrm{~K}$ & $\mathrm{R}$ & $S$ & $S$ & $\mathrm{~K}$ & $\mathrm{~T}$ & $\mathrm{Q}$ & 1 & A & $\mathrm{R}$ & $\mathrm{R}$ & $A$ \\
\hline ThNH36/93 & AFI69679 & DF & ASIAN I & 1 & V & 1 & $\mathrm{~K}$ & $\mathrm{R}$ & $\mathrm{S}$ & $S$ & $\mathrm{~K}$ & $\mathrm{~T}$ & $\mathrm{Q}$ & 1 & $\mathbf{A}$ & $\mathrm{R}$ & $\mathrm{R}$ & A \\
\hline ThNH45/93 & AFI69680 & DHF & ASIAN I & 1 & V & 1 & $\mathrm{~K}$ & $\mathrm{R}$ & $S$ & $S$ & $\mathrm{~K}$ & $\mathrm{~T}$ & $\mathrm{Q}$ & 1 & A & $\mathrm{R}$ & $\mathrm{R}$ & A \\
\hline
\end{tabular}


Table 3: Summary of consistent amino acid change among the five DENV-2 genotype viruses. (Continued)

\begin{tabular}{|c|c|c|c|c|c|c|c|c|c|c|c|c|c|c|c|c|c|c|}
\hline ThNH55/93 & AFI6968I & DHF & ASIAN I & $T$ & $\mathrm{~V}$ & $T$ & K & $R$ & $\mathrm{~S}$ & $\mathrm{~S}$ & $\mathrm{~K}$ & 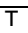 & $\mathrm{Q}$ & $T$ & $\mathbf{A}$ & $R$ & $\bar{R}$ & $\bar{A}$ \\
\hline ThNH54/93 & AFI69682 & DHF & ASIAN I & I & V & I & K & $\mathrm{R}$ & S & S & K & T & Q & 1 & A & $\mathrm{R}$ & $\mathrm{R}$ & A \\
\hline ThNH62/93 & AFI69683 & $\mathrm{DHF}$ & ASIAN I & I & V & I & K & $R$ & S & S & K & $\mathrm{T}$ & $\mathrm{Q}$ & I & $\mathbf{A}$ & $R$ & $R$ & A \\
\hline ThNH63/93 & AFI69684 & DHF & ASIAN I & 1 & $\mathrm{~V}$ & I & K & $\mathrm{R}$ & $\mathrm{S}$ & S & K & T & Q & 1 & A & $\mathrm{R}$ & $\mathrm{R}$ & A \\
\hline ThNH69/93 & AFI69685 & DHF & ASIAN I & 1 & V & I & K & $R$ & S & S & K & $\mathrm{T}$ & Q & I & A & $R$ & $R$ & A \\
\hline ThNH73/93 & AFI69686 & DHF & ASIAN I & 1 & V & I & K & $R$ & S & S & K & $\mathrm{T}$ & $\mathrm{Q}$ & 1 & $\mathbf{A}$ & $R$ & $R$ & A \\
\hline ThNH76/93 & AFI69687 & DHF & ASIAN I & 1 & V & I & K & $R$ & S & S & K & $\mathrm{T}$ & $\mathrm{Q}$ & I & $\mathbf{A}$ & $R$ & $R$ & A \\
\hline ThNH8I/93 & AFI69688 & $\mathrm{DHF}$ & ASIAN I & 1 & V & I & K & $R$ & S & S & K & $\mathrm{T}$ & $\mathrm{Q}$ & 1 & $\mathbf{A}$ & $R$ & $R$ & A \\
\hline K0008/Th/99 & AFI00459 & DHF & ASIAN I & 1 & V & I & K & $R$ & S & S & K & $\mathrm{T}$ & $\mathrm{Q}$ & 1 & $\mathbf{A}$ & $R$ & $R$ & A \\
\hline $\mathrm{K} 0010 / \mathrm{Th} / 99$ & $\mathrm{AFI} 00460$ & DF & ASIAN I & 1 & V & I & K & $\mathrm{R}$ & S & S & K & $\mathrm{T}$ & $\mathrm{Q}$ & 1 & $\mathbf{A}$ & $R$ & $R$ & A \\
\hline $\mathrm{C} 037 \mathrm{I} / \mathrm{Th} / 99$ & AFI0046I & DF & ASIAN I & 1 & V & I & K & $R$ & S & S & K & $\mathrm{T}$ & Q & 1 & $\mathbf{A}$ & $R$ & $R$ & A \\
\hline $\mathrm{C} 0166 / \mathrm{Th} / 99$ & AFI00463 & DF & ASIAN I & 1 & V & I & K & $\mathrm{R}$ & S & $S$ & K & $\mathrm{T}$ & Q & I & $\mathbf{A}$ & $R$ & $R$ & A \\
\hline $\begin{array}{l}\text { New Guinea C/ } \\
44\end{array}$ & D00346 & DHF & ASIAN 2 & 1 & V & I & K & $\mathrm{R}$ & S & $S$ & K & $\mathrm{T}$ & Q & I & $\mathrm{T}$ & $\mathrm{R}$ & $R$ & A \\
\hline 43/China/89 & AF204178 & DF & ASIAN 2 & 1 & V & I & K & $\mathrm{R}$ & $S$ & $S$ & K & $\mathrm{T}$ & Q & 1 & $\mathrm{~T}$ & $\mathrm{R}$ & $R$ & A \\
\hline 44/China/87 & AF204I77 & DF & ASIAN 2 & 1 & V & I & K & $R$ & S & S & K & $\mathrm{T}$ & $\mathrm{Q}$ & 1 & $\mathrm{~T}$ & $R$ & $R$ & A \\
\hline BA05i/Indon/05 & AY858035 & DF & COSMOPOLITAN & 1 & V & I & K & $\mathrm{R}$ & S & S & $\mathrm{T}$ & $\mathrm{T}$ & Q & 1 & $\mathrm{~T}$ & $\mathbf{K}$ & $R$ & A \\
\hline $\begin{array}{l}\text { TSVOI/Austra/ } \\
93\end{array}$ & AY037116 & NA & COSMOPOLITAN & 1 & V & I & K & $R$ & S & S & $\mathrm{T}$ & $\mathrm{T}$ & Q & I & $\mathrm{T}$ & $\mathbf{K}$ & $R$ & A \\
\hline $\begin{array}{l}98900666 / / n d o / \\
04\end{array}$ & $\mathrm{AB} 189124$ & DSS & COSMOPOLITAN & I & V & 1 & K & $\mathrm{R}$ & $\mathrm{S}$ & $\mathrm{S}$ & T & T & Q & I & $\mathrm{T}$ & $\mathbf{K}$ & $\mathrm{R}$ & A \\
\hline FJ-I0/China/00 & AF276619 & NA & COSMOPOLITAN & I & V & I & K & $\mathrm{R}$ & S & S & $\mathrm{R}$ & $\mathrm{T}$ & Q & I & $\mathrm{T}$ & K & $R$ & A \\
\hline
\end{tabular}

\&Amino acid sequence was numbered starting from protein NS5 or NS3 to the end of the last codon sequenced.

*Recombinant strain [16].

†NS3 Accession number for Oaxaca isolates: EF59|287-EF59| 293.

$\S$ Accession numbers for SAM/RdRp Domains

44 aa). In addition, phylogenetic analysis of $N S 3_{484}$ showed low variability $(1.9 \%, 2 / 103$ aa). Tables 2 and 3 summarizes the aa changes among the Asian I, Asian II, Cosmopolitan, American and American/Asian Genotype viruses. We compared the aas related to $\mathrm{C}_{91}-\mathrm{prM}_{1}, \mathrm{NS}_{75}$, $\mathrm{NS}_{2206}$ and $\mathrm{NS}_{484}$ in all Southeast Asian genotype viruses with American genotypes (Table 2 and 3). The former have greater potential to cause DHF while the latter are associated with DF $[18,25]$. The Oaxaca isolates in this study and all genotypes with potential to produce DHF contained valine at position 145 in C-prM (prM-31), except for the isolate S707OAX05/MEX, which contained threonine at this position as did in the American genotype. In addition, the Oaxaca isolates in this study and all genotypes with potential to produce DHF contained isoleucine, lysine and glutamine at positions 179, 192 and 818 respectively in NS5. In contrast, the American genotype had valine, arginine and leucine at these positions.

Frequency analysis included in addition to the sequences of Table 2, one sequence with Asian II genotype (M29095) and twelve with Cosmopolitan genotype (F276619, $\underline{\text { AF359579, }}$ AY858036, $\quad$ AB189122, AB189123， EF440433， DQ448233， DQ448234， DQ448235， DQ448236， DQ448237， AB111454). In addition to the sequences in Table 3, it was included 9 (EU570976, EU570977, EU570978, EU570979, EU570981， EU552540， EU552541， EU552542, EF595827) from Veracruz and 1 (EF595826) from Oaxaca in RdPd domain; and 1(EF591291) from Oaxaca in the Helicase domains.

Interestingly, the Oaxaca isolates in this study and the Caribbean islands strains contained valine at position 106 in $\mathrm{C}_{91}-\mathrm{prM}_{1}$ with a frequency of 0.65 in the Asian/Ameri- can genotype, while all other genotypes had isoleucine at this position (Table 2). All American/Asian viruses had valine, alanine and glycine at positions 104 (C), 112 (C) and 129 (prM-14), respectively, with a frequency of 1 , and isoleucine at position 153 (prM-39) with a frequency of 0.95 because HUAT2/MEX/01 had leucine at this position. The Cosmopolitan genotype had valine and alanine at positions 104 and 112 with a frequency of 0.67 and 0.61 , respectively (Table 2). American viruses had lysine and threonine at positions 142 (prM-28) and 145 (prM31 ) respectively, with a frequency of 1 . Three isolates from Oaxaca 2001 also contained lysine at position 142 (prM28 ), resulting in a frequency of 0.075 in the Asian/American genotype. Asian I genotype viruses had serine and isoleucine at positions 101 (C) and 130 (prM-15) with frequencies of 1 and 0.73 respectively. The other genotypes did not contain these amino acids. Cosmopolitan viruses had methionine and asparagine at positions 108 and 143 (prM-29) with a frequency of 0.44 in this genotype. The other genotypes did not contain this aa.

The American/Asian genotype viruses contained isoleucine, lysine, threonine, alanine and valine at positions $155,748,762,810$ and 835 in NS5 (Table 3 ) with a frequency of 1 for positions 155 and 748 and 0.96 for the positions 762, 810 and 835 (Table 3). Asian I contained the same aa at position 762 with a very low frequency of 0.035 in this genotype. Interestingly, our analysis showed that the Asian I genotype, unlike the others, had alanine at position 864 in NS5 rather than threonine with a frequency of 1 (Table 3). Furthermore, the American genotype had valine, arginine, serine and leucine with a frequency of 1 at positions 179, 192, 799 and 818 in NS5; none of the other genotypes has these aas at these positions, resulting in a frequency of 0 (Table 3 ). 


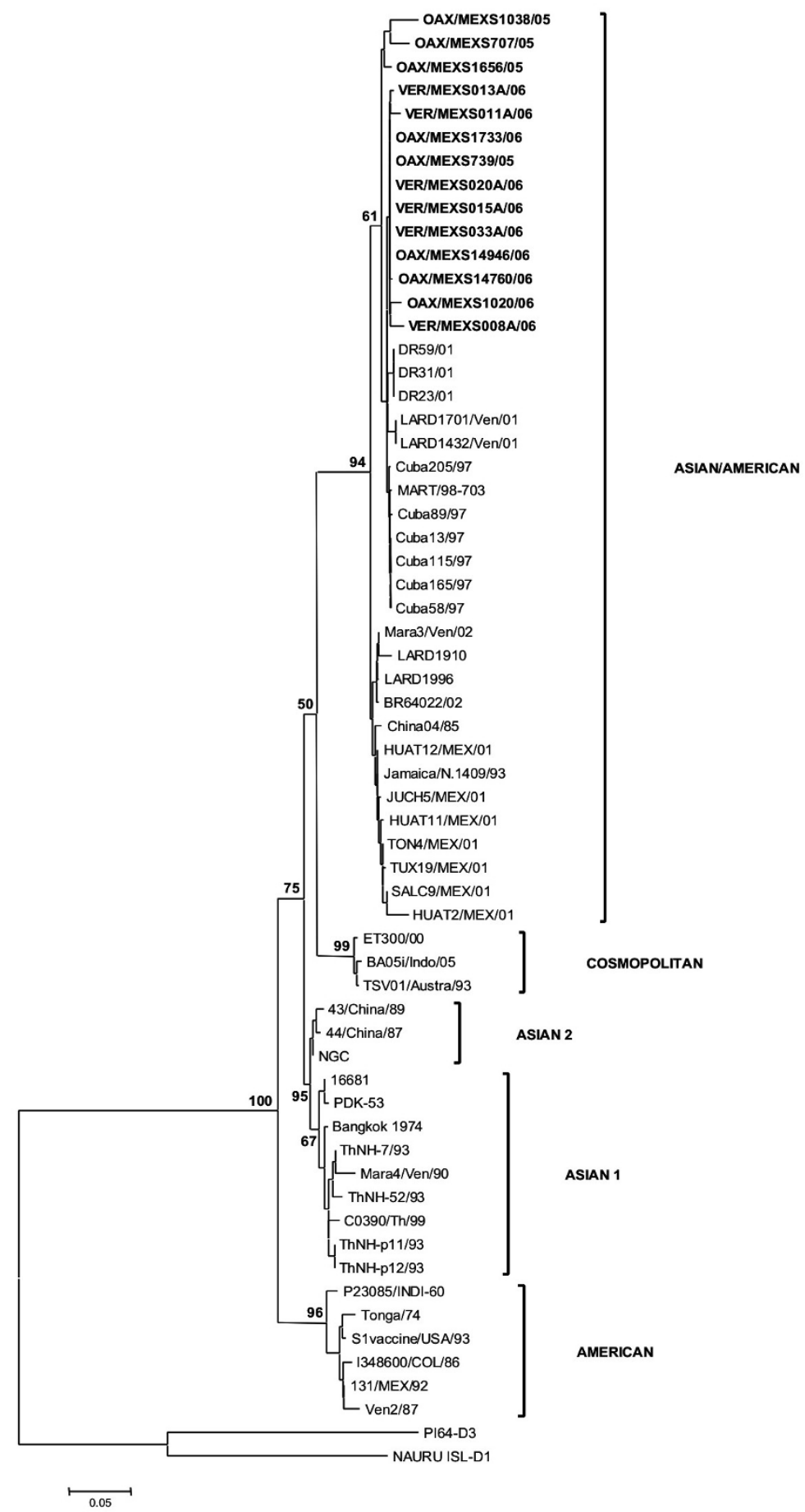

Figure 4

Minimal evolutionary tree of DENV-2 derived from the $\mathbf{C}_{9_{1}}-\mathbf{p r} \mathbf{M}_{1}$ gene. Branch lengths are proportional to percentage divergence. For distance/neighbor joining, maximum likelihood and Tamura Nei analyses, I000 bootstrap replications were performed with MEGA 4.0 [55]. The percentage of replications supporting each branch, by parsimony analysis with gaps included, appears below the relevant branch. Oaxaca isolates appear in bold in the Asian/American genotype cluster. DENV-I, 3 and 4 were used as outgroups. 


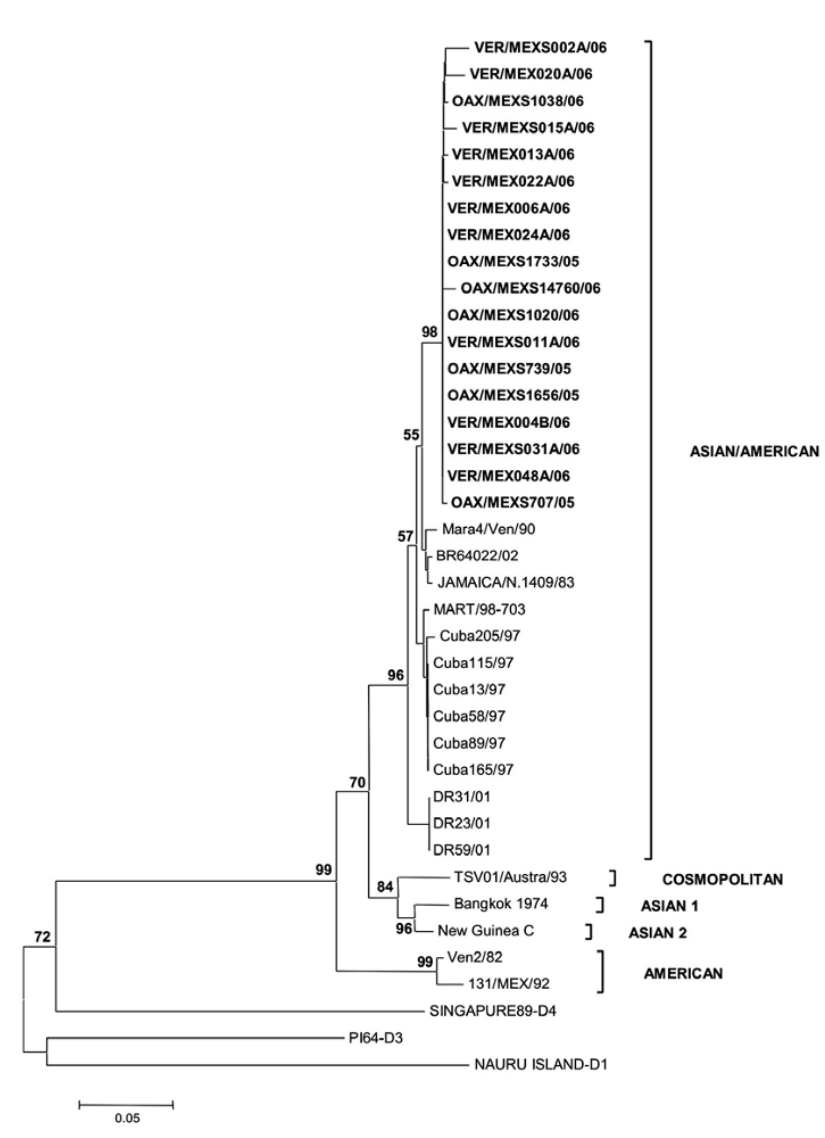

Figure 5

Minimal evolutional tree of DENV-2 derived from

NS5 $_{2206}$ gene. The tree was constructed as described in Figure 4. The isolates from Veracruz and Oaxaca clustered in the Asian/American genotype. DENV-I, 3 and 4 were used as outgroups.

American/Asian and Cosmopolitan genotypes had arginine at position 187 with a frequency of 1 in NS3 (Table 3). Oaxaca isolates had lysine and threonine at positions 249 and 250 in NS3 with frequencies of 0.33 and 0.38. Oaxaca and Veracruz isolates had valine and proline at positions 26 in NS5 and 784 in NS5 with frequencies of 0.33 and 0.56 respectively in the Asian/American genotype. Lysine at position 249 in NS3 may be signature of the Oaxaca strains, and valine and proline at positions 26 and 784 in NS5 may be signatures for the Oaxaca and Veracruz strains.

It is evident that the genotypically different strains contain specific aas that may be used as genotype markers.

\section{Discussion}

The Pan American Health Organization (PAHO) led a campaign to exterminate Ae. aegypti, which resulted in the eradication of YF and disappearance of DF in America. Ae. aegypti was absent from Mexico in 1947 until 1970. Because the mosquito was not completely exterminated in America and the campaigns were gradually relaxed, Ae. aegypti reappeared in most countries. There was a DENV1 outbreak in Mexico in 1978 and epidemics spread throughout the country. The first reports of outbreaks of DENV-2 were in 1981 and 1984, with 30,000 and 23,000 cases respectively. There were 8 reported DHF cases and 4 deaths associated with DENV-4 in Mexico in 1984. Four DHF cases and one death were reported in 1989 and 2 cases in 1991. DENV-1, -2 and -4 were isolated in 1994, and DENV-3 was reported in 1995. Furthermore, DENV$1,-2,-3$ and -4 were isolated in 1997 [34]. This data shows that even when DENV serotypes were co-circulating in Mexico, DHF cases were rare, suggesting that simultaneous circulation of several serotypes (hyperendemicity) is not necessarily sufficient to cause DHF epidemics in the absence of highly virulent strains, as previously reported [18]. Interestingly, the DENV-2 American genotype was replaced by the Asian/American genotype over this period. DF and DHF epidemics increased dramatically in the closing decades of the 20th century, especially in the New World, and Mexico was not excluded [34]. Furthermore, in south Texas (US), all dengue serotypes have circulated periodically, but locally acquired DHF has been recently reported [38]. The causes of this increased incidence of DENV infection, apart from the introduction of the DENV-2 Asian genotype, include demographic, cultural, environmental and political changes. This is clearly shown in Figure 1; DF and DHF cases have increased significantly since 2001 [34]. Furthermore, the proportion of DHF to DF cases has also increased (Figure 1), as in Thailand, where three DENV-2 genotypes have circulated but only viruses assigned to Asian genotype I have been sampled from the population since 1991 [25]. The genotype distribution in Mexico is mostly unknown; consequently, it is very important for epidemiological studies to determine the serotypes and genotypes of the viruses circulating in this country to contribute to the knowledge of geographic distribution, evolution and dispersion of DENV; besides, knowing the virus genotype may allow doctors to judge the risk of DHF to the patient. This may also be useful for laboratory diagnosis, which is an issue of serious concern in all endemic countries; diagnosis may well help in the rigorous follow-up of patients and could save them from the most severe DEN infection (DSS) and death.

Our results showed that DHF was associated with DENV1 and -2 and one case showed both serotypes (Table 1). Interestingly, we observed no DHF cases associated with DENV-3, as has been shown in epidemiological studies in Mexico [39]. Recently, DENV-3 has been isolated in Brazil but its association with DHF has not been clearly established [40]. In contrast, DENV-3 was associated with DHF 
in an outbreak in Cuba [13]. Phylogenetic analysis using portions of $C_{91}-p r M_{1}, N S 5_{75}, N S 5_{2206}$ and $N S 3_{484}$ showed no substantial differences (Figures 4,5 ).

In this study, we detected the amino acids glutamic acid and valine in place of lysine and threonine at positions 28 and $31 \mathrm{in} \mathrm{prM}$. These aas have been described as specific for the Asian strains correlated with DHF [12]. NS5 also contains lysine 46, arginine 47 and glutamic acid 49 in the MTase domain, essential for the interaction with RdRp required for virus replication and/or infectivity [28]. These three aas are conserved in all sequences of all strains or isolates.

We detected lysine and glutamine at positions 800 and 819 of NS5; these have been reported as markers of the Asian and Asian/American DENV-2 genotypes that have the potential to produce DHF [12]. The interdomain (linker) region 169-179 and the two loops that encircle the entrance of the ATP binding pocket of NS3 [31] were also studied because of the role of this protein in virus replication. This region has also been implicated in the intrinsic flexibility required to couple movements between the NS3 protease and helicase domains [31,32]. We found no change in these amino acids. However, at position 250 in the helicase domain, alanine (apolar) was replaced by threonine (polar) in the isolates in this study and in one strain from China (Table 3 ). It would seem important to study the structure of this region to determine whether this aa has any role in the enzymatic activity or in maintaining the protein structure.

The NS5 polymerase fragment (737 to 877 residues) contains conserved aas: cysteine at position 847 , which participates in the zinc pocket; threonine 790 to aspartic acid 808 and arginine 815 in the priming loop motif; and arginine 737, threonine 794, tryptophan 795 and serine 796, which are involved in GTP binding. However, the aa at position 810 in the priming loop is changed from threonine (polar) to alanine (apolar) in the Asian/American strains including our isolates (Table 3 ). It would seem important in the future to study the structure around this region to determine the function of this amino acid, particularly whether it has any role in polymerase activity [41].

A dramatic observation was obtained from the analysis of longitudinally sampled data on a temporal scale, where individual lineages or entire clades of viruses frequently arise, persist for a period of time and then disappear $[42,43]$. All strains analyzed in this work clustered in one branch with the Asian/American strains. Previously, in a survey during 2001 by the Department of Health $[17,33]$, we reported this genotype in isolates from Oaxaca on the basis of the protein $\mathrm{C}$ sequence and a fragment of the prM gene (438-572). The finding of the same Asian/American genotype in the isolates from the 2005 and 2006 outbreaks indicates that they circulated and became prevalent in Oaxaca and Veracruz States; however, the isolates in this study were grouped with the strains from the Caribbean islands, and the 2001 isolates with the Jamaica strain, with a high consistency of 94\%. Also, these 20052006 isolates from Oaxaca and Veracruz showed valine in position 106 in prM, which was also shown by the Caribbean islands strains. These isolates also clustered with the Caribbean islands strains Cuba 97, Dominican Republic 2001 and Martinique 98 in the phylogenetic analysis of $\mathrm{NS}_{75}$ and $\mathrm{NS}_{484}$ (results not shown), supporting this view of their geographic origin (Figure 2). All trees clearly distinguish among the five genotypes of DENV-2, as reported previously $[24,25]$, with strong bootstrap support (Figures 4,5). Little is known of the history of this American/Asian signature circulating in the Caribbean islands. However, Rodriguez-Roche et al. [44] found that clinical severity increased over time during the epidemics expressed by a higher ratio DHF to DF for the Cuban strains.

Apparently, there has been a clade replacement of the American genotype for the Asian/American genotype originated in Southeast Asia in Oaxaca (2001 outbreak) and lately that strains were substituted for the Oaxaca strains (2005-2006 outbreaks) with the probable origin in the Caribbean islands, possibly Cuba (1997 outbreak). Furthermore, the geographic origin of the Veracruz strains may be also the Caribbean islands.

Since analysis of the 98 C-prM sequences showed that all Asian/American viruses had valine, alanine, glycine and isoleucine at positions 129 (prM-14) and 153 (prM-39) with a frequency of 1 , while none of the other genotypes had these aas, they may be used as signatures of this genotype. The signature for American genotype viruses may be threonine at position 145 (prM-31) since none of the other genotypes had this amino acid. The signature for the Asian I genotype viruses may be serine at position 101 (C) with a frequency of 1 .

From the 65 (MTase) and 76 (RdRp) sequences analyzed, all Asian/American genotype viruses contained isoleucine, lysine, threonine, alanine, lysine and valine at positions 155, 748, 762, 810 and 835 (Table 3) and none of the other genotypes had these aas. Our analysis also showed that the Asian I genotype, unlike the others, had alanine at position 864 (RdRp). The American genotype had valine, arginine, serine and leucine with frequency of 1 at positions179, 192, 799 and 818, but none of the DENV-2 genotypes had it. All aas specific for a genotype may be used as signatures for that genotype. Although fewer sequences were analyzed than for C-prM, because 
these genes have been less studied and consequently fewer sequences have been reported. The Asian 2 and Cosmopolitan genotypes showed no specific signature in the present study. This may be attributable to the broad spectrum of the Cosmopolitan genotype strains and the low number of Asian 2 sequences reported.

Lysine at position 249 in NS3 may be signature of the Oaxaca strains, and valine and proline at positions 26 and 784 in NS5 may be signatures for the Oaxaca and Veracruz strains, since none of the other genotypes or strains from the Asian/American genotype had these aas.

Some of the observed changes in the viruses could be due to mosquito adaptation as well as to the vertebrate hosts, since there is evidence that strains of DENV-2 may differ in their ability to infect Ae. aegypti [45], perhaps through specific virus-cell receptor interactions $[46,47]$ by strong selection, or competition between DENV serotypes [48]. It has been suggested that the Asian genotype may spread more readily because it infects Ae. aegypti mosquitoes more efficiently than the American genotype [45].

The amino acid changes between the isolates of this study and those from 2001 may be due to a high mutation rate [49] or recombination [50] contributing to the generation of new biologically successful strains.

Phylogenetic analysis of the $\mathrm{C}_{91}-\mathrm{prM}_{1}, \mathrm{NS}_{75}, \mathrm{NS}_{2206}$ and $\mathrm{NS}_{484}$ sequences in the DENV-2 isolates demonstrated that the Asian/American genotype is circulating in Oaxaca and Veracruz, showing specific amino acid changes that may be used successfully as genotype markers. Differences in the $\mathrm{NS5}_{2206}$ tree, which showed that the isolates in this study clustered with the strains from the contained American genotype, may be due to the low variability of this domain. In addition, this analysis shows a clear correlation between genetic diversity and geographical location. As has been suggested previously $[43,51]$, viral dispersion may play a significant role in generating new varieties and effects on people who contract the illness.

\section{Conclusion}

We have demonstrated that DENV-1, -2 and -3 are circulating in Oaxaca and DENV-1, $-2,-3$ and -4 in Veracruz (2006), resulting in many DHF cases (Figure 1, 3). Eight DHF cases were associated with DENV-1, 27 with DENV2 and one case with both DENV-1 and -2. The origin of the isolates in this study appears to be linked to the signature from the Caribbean islands genotypes. Our results confirm that DHF is endemic in Oaxaca and Veracruz States and that the presence of the DENV-2 Asian/American genotype and DENV-1 contributes to the increased number of DHF cases, a finding linked to the epidemiological data showing an increase in the ratio of DHF to DF cases in
2005-2006. We also identified amino acids encoded in $\mathrm{C}_{91}-\mathrm{prM}_{1}, \mathrm{NS}_{75}, \mathrm{NS}_{2206}$ and $\mathrm{NS}_{484}$ that are specific to the Asian/American strains; and one amino acid specific to the isolates of this study and the Caribbean islands strains. These aas may be used as markers of genotypes and geographic origins.

\section{Methods \\ Viruses}

The strains of DENV used in this study were: DENV-1 Hawaii; DENV-2 New Guinea C (NGC); DENV-3 H-87; and DENV-4 H-341. These strains were provided by Dr. D. J. Gubler (Division of Vector-borne Infectious Diseases, Center for Disease Control, Fort Collins, CO, USA) and kindly provided by Dr. Blanca Ruiz (Biomedicas, UNAM). Isolates of DENV-2 viruses were obtained from acutephase plasma collected from patients with DF or DHF by the Secretaria de Salud in Oaxaca, Mexico during 20052006, and by the Instituto Mexicano del Seguro Social (IMSS) during 2006. Twenty-nine isolates were kindly provided by the State of Oaxaca Public Health Laboratory and 65 by the IMSS in Veracruz. These were anonymous samples; only information about the clinical disease associated with the respective infection was provided (Tables $1)$.

\section{DENV infected cells and virus isolation}

Ae. albopictus clone $\mathrm{C} 6 / 36$ cells were grown at $28^{\circ} \mathrm{C}$. After $18 \mathrm{~h}$ of culture, cells $\left(2 \times 10^{6} / 100 \mathrm{~mm}\right.$ plate $)$ were infected with $0.2 \mathrm{ml}$ DENV-2 inoculum with an input MOI of 600 $\mathrm{PFU} /$ cell and were incubated at $28^{\circ} \mathrm{C}$ for 10 days.

Viruses were isolated as previously described [17] with a few modifications. After $18 \mathrm{~h}$ of culture, C6/36 cells (10\% $15 \mathrm{ml}$ tube) were infected with 0.01 to $0.1 \mathrm{ml}$ of serum specimen per tube, diluted to $1 \mathrm{ml}$ with medium, and incubated for $2 \mathrm{~h}$ at $28^{\circ} \mathrm{C}$. After one wash, $3 \mathrm{ml} \mathrm{MEM}$ was added and the cells were cultivated for approximately 15 days at $28^{\circ} \mathrm{C}$ (passage number 1). Cells were observed every day and when a cytophatic effect was apparent from syncytium formation and cellular lysis, the cells were harvested and centrifuged at $3000 \mathrm{rpm}$ for $5 \mathrm{~min}$. The pellet was suspended in $0.6 \mathrm{ml}$ of MEM and stored in aliquots of $0.15 \mathrm{ml}$ at $-70^{\circ} \mathrm{C}$. The supernatant (approximately $2.5 \mathrm{ml}$ ) was stored in 2 aliquots of $1 \mathrm{ml}$ and one of $0.5 \mathrm{ml}$ at $70^{\circ} \mathrm{C}$. To obtain passage numbers two and three, C6/36 cells were incubated with $1 \mathrm{ml}$ of the supernatant obtained from the first or second passage for $2 \mathrm{~h}$ at $28^{\circ} \mathrm{C}$ and the same procedure described above was followed. Serotypes in all samples were determined in the isolates obtained from the first, second or third culture passages.

\section{RNA extraction}

Total RNA was extracted from cell culture supernatant using Trisol ${ }^{\varpi}$ LS (Gibco BRL., Gaithersburg, Md.) accord- 
ing to the manufacturer's recommendations. Ethanol-precipitated RNA was recovered by centrifugation and airdried. The RNA pellet was suspended in $50 \mu \mathrm{l}$ water treated with diethylpyrocarbonate (DEPC, Sigma-Aldrich) and used as template for Reverse Transcription with the Polymerase Chain Reaction (RT-PCR).

\section{Reverse transcription-polymerase chain reaction (RT- PCR)}

The RT-PCR protocol described by Seah et al. [52] was followed to determine the DENV serotype. Reaction mixtures were stored at $-20^{\circ} \mathrm{C}$ pending further processing.

Synthetic oligonucleotide primer pairs were designed on the basis of published sequence data for DENV strains 16681, New Guinea C and Jamaica 1409 to amplify and partially sequence the following genes: protein $C$ from nucleotides 91 to $351\left(\mathrm{C}_{91}\right) ; \operatorname{prM}$ from nucleotides 1 to $123\left(\mathrm{prM}_{1}\right)$ [17]; NS5 from nucleotides 73 to $588\left(\mathrm{NS5}_{73}\right)$ and 2206 to 2613 (NS5 2206 ); and NS3 from nucleotides 484 to $786\left(\mathrm{NS}_{484}\right)$. All assays were performed using the ThermoScript ${ }^{\mathrm{TM}}$ RT-PCR System containing PlatinumR Taq (Invitrogen, Life Technologies). A mixture of $5 \mu \mathrm{l}$ total RNA $(0.1-0.5 \mu \mathrm{g}), 50 \mathrm{ng}$ of hexamers/reaction and DEPCtreated water (total volume $50 \mu \mathrm{l}$ ) was incubated at $85^{\circ} \mathrm{C}$ for $5 \mathrm{~min}$ and chilled on ice. The first extension was carried out at $25^{\circ} \mathrm{C}$ for $10 \mathrm{~min}$ and then at $50^{\circ} \mathrm{C}$ for $70 \mathrm{~min}$. The PCR reaction was carried out by incubation of 50 pmol of the corresponding sense and antisense PCR primers, $2 \mu \mathrm{l}$ of the cDNA synthesis reaction mixture and 2.4 $\mathrm{mM}$ magnesium sulfate as per the manufacturer's recommendations.

To amplify the NS5 gene we used the sense primer from nucleotides 7398-7413 (CAT GGG CNY TNT GYGA) and the antisense primer from nucleotides 10551-10570 (GGA GGG GTC TCC TCT AACC). The PCR conditions for amplifying the $2700 \mathrm{bp}$ fragment for NS5 were: $95^{\circ} \mathrm{C}$ for 5 min, 30 cycles of $94^{\circ} \mathrm{C}$ for $1 \mathrm{~min}, 49^{\circ} \mathrm{C}$ for $30 \mathrm{~s}, 72^{\circ} \mathrm{C}$ for $2 \mathrm{~min} 30 \mathrm{~s}$, and final extension of $72^{\circ} \mathrm{C}$ for $10 \mathrm{~min}$ followed by storage at $4{ }^{\circ} \mathrm{C}$.

\section{Sequencing of PCR fragments}

For automated sequencing, spin column-purified (Quiagen, Chatsworth, CA.) DNA fragments were analyzed by the cycle-sequencing dye terminator method. A Big Dye Terminator Cycle Sequencing Ready Reaction Kit (ABIPRISM 100, Applied Biosystems, Foster City, CA) was chosen and sequencing was conducted using an Applied Biosystems Prism 310 in a short capillary $(47 \mathrm{~cm}$ by 50 $\mu \mathrm{m}$ inside diameter), and Performance Optimized Polymer 6 (Perkin-Elmer, Applied Biosystems).

\section{Phylogenetic analyses}

Neighbor-joining (NJ), maximum parsimony and minimal evolutionary [53] analysis methods were used to reconstruct phylogeny. Distance with gamma correction was generated by Neighbor-joining, maximum likelihood and Tamura and Nei [54] evolutionary models. Support for the derived phylogenies was examined with bootstrapping over 1000 replications. For all DENV-2 analyses, DENV-1, -3 and -4 were used as outgroups. All these analyses were performed using MEGA V4 [55].

\section{Authors' contributions}

CEGG carried out the RT-PCR assays using RNA isolates from passages 1, 2 and 3 to determine serotype and to amplify the NS5 and NS3 genes for genotyping, and cowrote the manuscript. GPR obtained the isolates and carried out the RT-PCR assays using RNA from passages 1, 2 or 3 to sequence the C-prM gene for genotyping by phylogenetic analysis. CEGG, GPR and MLM participated in the discussion of results, helped to review the manuscript and assisted with the literature validation. JNE and RRL provided the serum samples from Veracruz and the clinical data corresponding to each patient, and helped in obtaining the isolates. FJR, LRRP and AC collected the patient serum samples from Oaxaca, Mexico, and helped to obtain the isolates and clinical data from Oaxaca. MCN participated in the discussion of results and reviewed the manuscript. MLM proof-read and assembled the manuscript. All authors participated in the discussion of results and read and approved the final manuscript.

\section{Acknowledgements}

Rosalinda Tovar and Dr. Alvaro Diaz-Badillo are gratefully acknowledged for their help during the development of this research. This work was supported by the CONACYT grant CB-2005-0I-50603 and CONACYTCOVECYT M0034-2007-68I23.

\section{References}

I. Garcia-Franco F, Munoz ML, Lozano-Fuentes S, Fernandez-Salas I, Garcia-Rejon J, Beaty BJ, Black WC 4th: Large genetic distances among Aedes aegypti populations along the South Pacific coast of Mexico. Am J Trop Med Hyg 2002, 66(5):594-598.

2. Gorrochotegui-Escalante N, Munoz ML, Fernandez-Salas I, Beaty BJ, Black WC 4th: Genetic isolation by distance among Aedes aegypti populations along the northeastern coast of Mexico. Am J Trop Med Hyg 2000, 62(2):200-209.

3. Gorrochotegui-Escalante N, Gomez-Machorro C, Lozano-Fuentes S, Fernandez-Salas L, Munoz ML, Farfan-Ale JA, Garcia-Rejon J, Beaty BJ, Black WC 4th: Breeding structure of Aedes aegypti populations in Mexico varies by region. Am J Trop Med Hyg 2002, 66(2):213-222.

4. Gubler DJ: Dengue and dengue hemorrhagic fever. Clin Microbiol Rev 1998, II (3):480-496.

5. WHO (World Health Organization): Dengue haemorrhagic fever: early recognition, diagnosis and hospital management - an audiovisual guide for health care workers responding to outbreaks. Weekly Epidemiological Record 2006, 8 I (38):362-363.

6. Clinical diagnosis Dengue haemorrhagic fever: diagnosis, treatment, prevention and control 2nd edition. 1997:12-23 [http://www.who.int/ csr/resources/publications/dengue/Denguepublication/en/]. World Health Organization. Geneva 
7. Navarrete-Espinosa J, Gomez-Dantes H, Celis-Quintal JG, VazquezMartinez JL: Clinical profile of dengue hemorrhagic fever cases in Mexico. Salud Publica Mex 2005, 47:193-200.

8. Rothman AL: Immunology and immunopathogenesis of dengue disease. Adv Virus Res 2003, 60:397-419.

9. Guzman MG, Alvarez M, Rodriguez-Roche R, Bernardo L, Montes T, Vazquez S, Morier L, Alvarez A, Gould EA, Kouri G, Halstead SB: Neutralizing antibodies after infection with dengue I virus. Emerg Infect Dis 2007, I3(2):282-286.

10. Wang WK, Chao DY, Lin SR, King CC, Chang SC: Concurrent infections by two dengue virus serotypes among dengue patients in Taiwan. I Microbiol Immunol Infect 2003, 36(2):89-95.

II. Rico-Hesse R, Harrison LM, Salas RA, Tovar D, Nisalak A, Ramos C, Boshell J, de Mesa MT, Nogueira RM, da Rosa AT: Origins of dengue type 2 viruses associated with increased pathogenicity in the Americas. Virology 1997, 230(2):244-25I.

12. Leitmeyer KC, Vaughn DW, Watts DM, Salas R, Villalobos I, de Chacon, Ramos C, Rico-Hesse R: Dengue virus structural differences that correlate with pathogenesis. J Virol 1999, 73(6):4738-4747.

13. Alvarez M, Rodriguez-Roche R, Bernardo L, Vazquez S, Morier L, Gonzalez D, Castro O, Kouri G, Halstead SB, Guzman MG: Dengue hemorrhagic fever caused by sequential dengue I-3 virus infections over a long time interval: Havana epidemic, 200 I2002. Am J Trop Med Hyg 2006, 75(6): III3-III7.

14. Thein S, Aung MM, Shwe TN, Aye M, Zaw A, Aye K, Aye KM, Aaskov J: Risk factors in dengue shock syndrome. Am J Trop Med Hyg | 997, 56(5):566-572.

15. Guzman MG, Deubel V, Pelegrino JL, Rosario D, Marrero M, Sariol C, Kouri G: Partial nucleotide and amino acid sequences of the envelope and the envelope/nonstructural protein-I gene junction of four dengue-2 virus strains isolated during the I 98 I Cuban epidemic. Am J Trop Med Hyg 1995, 52(3):24|-246.

16. Uzcategui NY, Camacho D, Comach G, Cuello de Uzcategui R, Holmes EC, Gould EA: Molecular epidemiology of dengue type 2 virus in Venezuela: evidence for in situ virus evolution and recombination. J Gen Virol 200I, 82(Pt I 2):2945-2953.

17. Cisneros A, Diaz-Badillo A, Cruz-Martinez G, Tovar R, Ramirez-Palacios LR, Jimenez-Rojas F, Beaty B, Black WC 4th, Munoz LM: Dengue 2 genotypes in the state of Oaxaca, Mexico. Arch Viro 2006, I 5 I(I): I I3-I25.

18. Watts DM, Porter KR, Putvatana P, Vasquez B, Calampa C, Hayes CG, Halstead SB: Failure of secondary infection with American genotype dengue 2 to cause dengue haemorrhagic fever. Lancet |999, 354(9|88): |43|-|434.

19. Lanciotti RS, Lewis JG, Gubler DJ, Trent DW: Molecular evolution and epidemiology of dengue-3 viruses. J Gen Virol 1994, 75(Pt I):65-75.

20. Lanciotti RS, Gubler DJ, Trent DW: Molecular evolution and phylogeny of dengue-4 viruses. J Gen Virol I997, 78(Pt 9):2279-2284.

21. Lewis JA, Chang G], Lanciotti RS, Kinney RM, Mayer LW, Trent DW: Phylogenetic relationships of dengue-2 viruses. Virology 1993, I97(I):216-224

22. Rico-Hesse R: Molecular evolution and distribution of dengue viruses type I and 2 in nature. Virology I990, I 74(2):479-493.

23. Twiddy SS, Farrar Jן, Vinh Chau N, Wills B, Gould EA, Gritsun T, Lloyd G, Holmes EC: Phylogenetic relationships and differential selection pressures among genotypes of dengue-2 virus. Virology 2002, 298(I):63-72.

24. Holmes EC, Twiddy SS: The origin, emergence and evolutionary genetics of dengue virus. Infect Genet Evol 2003, 3(I):19-28.

25. Zhang C, Mammen MP, Chinnawirotpisan P, Klungthong C, Rodpradit P, Nisalak A, Vaughn DW, Nimmannitya S, Kalayanarooj S, Holmes EC: Structure and age of genetic diversity of dengue virus type 2 in Thailand. J Gen Virol 2006, 87(Pt 4):873-883.

26. Chua SK, Selvanesan S, Sivalingam B, Chem YK, Norizah I, Zuridah H, Kumarasamy $\mathrm{V}$, Chua KB: Isolation of monoclonal antibodiesescape variant of dengue virus serotype I. Singapore Med J 2006, 47( I I):940-946.

27. Rodriguez-Roche R, Alvarez M, Gritsun T, Rosario D, Halstead S, Kouri G, Gould EA, Guzman MG: Dengue virus type 2 in Cuba, 1997: conservation of $E$ gene sequence in isolates obtained at different times during the epidemic. Arch Virol 2005 I 50(3):415-425

28. Malet H, Egloff MP, Selisko B, Butcher RE, Wright PJ, Roberts M, Gruez A, Sulzenbacher G, Vonrhein C, Bricogne G, Mackenzie JM,
Khromykh AA, Davidson AD, Canard B: Crystal structure of the RNA polymerase domain of the West Nile virus non-structural protein 5. J Biol Chem 2007, 282(14): 10678-10689.

29. Kuno G, Chang GJ, Tsuchiya KR, Karabatsos N, Cropp CB: Phylogeny of the genus Flavivirus. J Virol 1998, 72(I):73-83.

30. Baleotti FG, Moreli ML, Figueiredo LT: Brazilian Flavivirus phylogeny based on NS5. Mem Inst Oswaldo Cruz 2003, 98(3):379-382.

31. Luo D, Xu T, Hunke C, Grüber G, Vasudevan SG, Lescar J: Crystal structure of the NS3 protease-helicase from dengue virus. Virol 2008, 82(I): I73-183.

32. Rosales-Leon L, Ortega-Lule G, Ruiz-Ordaz B: Analysis of the domain interactions between the protease and helicase of NS3 in dengue and hepatitis C virus. J Mol Graph Model 2007, 25(5):585-594.

33. Cisneros-Solano A, Moreno-Altamirano MM, Martinez-Soriano $U$, Jimenez-Rojas F, Diaz-Badillo A, Munoz ML: Sero-epidemiological and virological investigation of dengue infection in Oaxaca, Mexico, during 2000-200 I. Dengue Bulletin 2004, 28:28-34.

34. DGEPI (Dirección General de Epidemiologia de la Secretaria de Salud) 200I-2006 Boletin Epidemiolgia [http:// www.dgepi.salud.gob.mx/boletin/].

35. Chen WJ, Wu HR, Chiou SS: E/NSI modifications of dengue 2 virus after serial passages in mammalian and/or mosquito cells. Intervirology 2003, 46(5):289-295.

36. Lee $E$, Weir RC, Dalgarno L: Changes in the dengue virus major envelope protein on passaging and their localization on the three-dimensional structure of the protein. Virology 1997, 232(2):28I-290.

37. Twiddy SS, Woelk CH, Holmes EC: Phylogenetic evidence for adaptive evolution of dengue viruses in nature. J Gen Virol 2002, 83(Pt 7): |679-|689.

38. Ramos MM, Mohammed H, Zielinski-Gutierrez E, Hayden MH, Lopez JL, Fournier M, Trujillo AR, Burton R, Brunkard JM, Anaya-Lopez L, Banicki AA, Morales PK, Smith B, Munoz JL, Waterman SH, Dengue Serosurvey Working Group: Epidemic dengue and dengue hemorrhagic fever at the Texas-Mexico border: Results of a household-based seroepidemiologic survey, December 2005. Am J Trop Med Hyg 2008, 78(3):364-369.

39. Manual para la Vigilancia, Diagnostico, Prevencion y Control del Dengue [http://salud.tamaulipas.gob.mx/epidemiologia/pdf's/ MANUAL-DENGUE\%202006.pdf

40. Pires Neto RJ, Lima DM, de Paula SO, Lima CM, Rocco IM, Fonseca $B A$ : Molecular epidemiology of type $I$ and 2 dengue viruses in Brazil from 1988 to 200I. Braz J Med Biol Res 2005, 38(6):843-852

4I. Yap TL, Xu T, Chen YL, Malet H, Egloff MP, Canard B, Vasudevan SG, Lescar J: Crystal structure of the dengue virus RNA-dependent RNA polymerase catalytic domain at 1.85 -angstrom resolution. J Virol 2007, 8 I(9):4753-4765.

42. Wittke $V$, Robb TE, Thu HM, Nisalak A, Nimmannitya S, Kalayanrooj S, Vaughn DW, Endy TP, Holmes EC, Aaskov JG: Extinction and rapid emergence of strains of dengue 3 virus during an interepidemic period. Virology 2002, 30 I(I): | 48-I56.

43. Zhang C, Mammen MP Jr, Chinnawirotpisan P, Klungthong C, Rodpradit P, Monkongdee P, Nimmannitya S, Kalayanarooj S, Holmes EC: Clade replacements in dengue virus serotypes $I$ and 3 are associated with changing serotype prevalence. J Virol 2005, 79(24): $15123-15130$

44. Rodriguez-Roche R, Alvarez M, Gritsun T, Halstead S, Kouri G, Gould $E A$, Guzman MG: Virus evolution during a severe dengue epidemic in Cuba, 1997. Virology 2005, 334(2): I54-I59.

45. Armstrong PM, Rico-Hesse R: Efficiency of dengue serotype 2 virus strains to infect and disseminate in Aedes aegypti. Am J Trop Med Hyg 2003, 68(5):539-544.

46. Mercado-Curiel RF, Esquinca-Aviles HA, Tovar R, Diaz-Badillo A Camacho-Nuez M, Munoz ML: The four serotypes of dengue recognize the same putative receptors in Aedes aegypti midgut and Ae. albopictus cells. BMC Microbiol 2006, 6:85.

47. Munoz ML, Cisneros A, Cruz J, Das P, Tovar R, Ortega A: Putative dengue virus receptors from mosquito cells. FEMS Microbiol Lett 1998, I 68(2):251-258.

48. Pepin KM, Lambeth K, Hanley KA: Asymmetric competitive suppression between strains of dengue virus. BMC Microbiol 2008 , 8:28.

49. Drake JW: Rates of spontaneous mutation among RNA viruses. Proc Natl Acad Sci USA 1993, 90(9):4I7I-4I75. 
50. Worobey M, Rambaut A, Holmes EC: Widespread intra-serotype recombination in natural populations of dengue virus. Proc Natl Acad Sci USA 1999, 96( (13):7352-7357.

5I. Bennett SN, Holmes EC, Chirivella M, Rodriguez DM, Beltran M, Vorndam V, Gubler DJ, McMillan WO: Selection-driven evolution of emergent dengue virus. Mol Biol Evol 2003, 20(I 0): 1650-1658.

52. Seah CL, Chow VT, Tan HC, Can YC: Rapid, single-step RT-PCR typing of dengue viruses using five NS3 gene primers. J Virol Methods 1995, 5 I(2-3): 193-200.

53. Saitou N, Nei M: The neighbor-joining method: a new method for reconstructing phylogenetic trees. Mol Biol Evol 1987, 4(4):406-425.

54. Tamura K, Nei M: Estimation of the number of nucleotide substitutions in the control region of mitochondrial DNA in humans and chimpanzees. Mol Biol Evol 1993, I0(3):5 I 2-526.

55. Tamura K, Dudley J, Nei M, Kumar S: MEGA4: Molecular Evolutionary Genetics Analysis (MEGA) software version 4.0. Mol Biol Evol 2007, 24(8): I596-I599.

Publish with Bio Med Central and every scientist can read your work free of charge

"BioMed Central will be the most significant development for disseminating the results of biomedical research in our lifetime. "

Sir Paul Nurse, Cancer Research UK

Your research papers will be:

- available free of charge to the entire biomedical community

- peer reviewed and published immediately upon acceptance

- cited in PubMed and archived on PubMed Central

- yours - you keep the copyright

Submit your manuscript here:

http://www.biomedcentral.com/info/publishing_adv.asp
BiolMedcentral 